\title{
Free fatty acid receptor 4 is a nutrient sensor that resolves inflammation to maintain cardiac homeostasis
}

Katherine A. Murphy, $\mathrm{PhD}^{1}$, Brian A. Harsch, $\mathrm{MS}^{2}$, Chastity L. Healy, BS ${ }^{1}$, Sonal S. Joshi, $\mathrm{PhD}^{1}$, Shue Huang, $\mathrm{MS}^{2}$, Rachel E. Walker, $\mathrm{PhD}^{2}$, Brandon M. Wagner, BA ${ }^{1}$, Katherine M. Ernste, $\mathrm{BA}^{1}$, Wei Huang, $\mathrm{MS}^{3}$, Robert Block, $\mathrm{MD}^{4}$, Casey D. Wright, $\mathrm{PhD}^{5}$, Nathan Tintle, $\mathrm{PhD}^{6}$, Brian C. Jensen, $\mathrm{MD}^{3}$, Gregory C, Shearer, $\mathrm{PhD}^{2,7}$, and Timothy D. O'Connell, $\mathrm{PhD}^{1,7}$.

${ }^{1}$ Department of Integrative Biology and Physiology, University of Minnesota, Minneapolis, MN

${ }^{2}$ Department of Nutritional Sciences, Pennsylvania State University, University Park, PA

${ }^{3}$ Division of Cariology and McCallister Heart Institute, University of North Carolina, Chapel Hill, NC

${ }^{4}$ Department of Public Health Sciences, University of Rochester, NY

${ }^{5}$ Current address, Inanovate Inc., Sioux Falls, SD

${ }^{6}$ Department of Statistics, Dordt University, Sioux Center, IA

${ }^{7}$ Correspondence to:

Timothy D. O'Connell, PhD

Department of Integrative Biology and Physiology

University of Minnesota School of Medicine

3-141 CCRB

2231 6th Street SE

Minneapolis, MN 55414

Email: tdoconne@umn.edu

Gregory C Shearer, PhD

Department of Nutritional Sciences

110 Chandlee Laboratory

University Park, PA 16802

gcs13@psu.edu 


\section{Abstract (247)}

Background: Non-resolving activation of immune responses is central to the pathogenesis of heart failure (HF). Free fatty acid receptor 4 (Ffar4) is a G-protein coupled receptor (GPR) for medium- and long-chain fatty acids (FA) that regulates metabolism and attenuates inflammation in diabetes and obesity. Here, we tested the hypothesis that Ffar4 functions as a cardioprotective nutrient sensor that resolves inflammation to maintain cardiac homeostasis.

Methods: Mice with systemic deletion of Ffar4 (Ffar4KO) were subjected to pressure overload by transverse aortic constriction (TAC). Transcriptome analysis of cardiac myocytes was performed three days post-TAC. Additionally, Ffar4-mediated effects on inflammatory oxylipin production in cardiac myocytes and oxylipin composition in plasma lipoproteins were evaluated. Results: In Ffar4KO mice, TAC induced more severe remodeling, identifying an entirely novel cardioprotective role for Ffar4 in the heart. Transcriptome analysis 3-days post-TAC indicated a failure to induce cell death and inflammatory genes in Ffar4KO cardiac myocytes, as well as a specific failure to induce cytoplasmic phospholipase $A_{2} \alpha\left(C P L A_{2} \alpha\right)$ signaling genes. In cardiac myocytes, Ffar4 signaling through cPLA $2 \alpha$-cytochrome p450 $\omega / \omega-1$ hydroxylase induced production of the EPA-derived anti-inflammatory oxylipin 18-hydroxyeicosapentaenoic acid (18HEPE). Systemically, loss of Ffar4 altered oxylipin content in circulating plasma lipoproteins consistent with a loss of anti-inflammatory oxylipins at baseline, and inability to produce both pro-inflammatory and pro-resolving oxylipins following TAC. Finally, we confirmed that Ffar4 is expressed in human heart and down-regulated in HF.

Conclusions: Our results identify a novel function for Ffar4 in the heart as a FA nutrient sensor that resolves inflammation to maintain cardiac homeostasis.

Keywords: Free fatty acid receptor 4 (Ffar4), GPR120, heart failure, cytoplasmic phospholipase $\mathrm{A}_{2} \alpha$ (cPLA $2 \alpha$ ), 18-hydroxyeicosapentaenoic acid (18-HEPE) 


\section{Introduction (777)}

Heart failure (HF) is a complex and heterogeneous clinical syndrome, and in the last 10 years, two broadly defined phenotypes have emerged: HF with reduced ejection fraction (HFrEF) and HF with preserved ejection fraction (HFpEF). It is now clear that failure to resolve immune activation is central to the pathogenesis of HF, both HFrEF and HFpEF. In HFrEF, typically observed secondary to ischemic injury, cardiac cell death drives an initial immune response to induce scar formation, but failure to resolve inflammation can worsen long-term ventricular remodeling (Review: (1)). In HFpEF, it has been proposed that comorbidities associated with metabolic syndrome induce peripheral inflammation that drives remodeling (Reviews (2-4)). Clinically, targeting the innate immune response has shown some success. The Canakinumab Anti-inflammatory Thrombosis Outcome Study (CANTOS) trial demonstrated

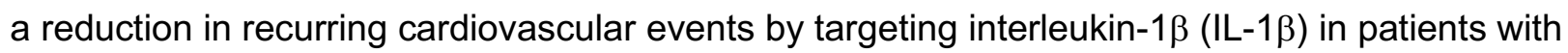
a prior myocardial infarction and high C-reactive protein (5). While the results of CANTOS are promising, successes targeting immune modulators have not been universal (6), suggesting the need for better understanding of the immune response in HF and the identification new therapeutic targets.

Free fatty acid receptor 4 (Ffar4, GPR120) is a G-protein coupled receptor (GPR) that functions as a nutrient sensor for fatty acids (FA) to regulate metabolism and attenuate inflammation $(7,8)$. The endogenous ligands for Ffar4 include medium and long chain (C10C22), saturated (SFA), mono-unsaturated (MUFA), and poly-unsaturated fatty acids (PUFA), which bind the receptor with affinities in the low $\mu \mathrm{M}$ range. In terms of agonist efficacy, generally, PUFAs are full agonists, whereas SFAs are partial agonists (9-11). Consistent with a primary role in regulating metabolism, Ffar4 is expressed in enteroendocrine cells in the GI tract (11), $\alpha, \beta$, and $\delta$-cells in the pancreas (11-13), and both white and brown adipose $(14,15)$. However, Ffar4 is found in many additional tissues with high levels of expression in the lung, and lower levels of expression in brain, heart, taste buds, and immune cells, including macrophages (10, 16-18). Ffar4 signals through both $\mathrm{G}_{\mathrm{q} / 11}$ and $\beta$ arrestin-2 ( $\beta$ Arr2) mediated pathways, which appears to be cell-type dependent $(10,17,19,20)$. Interestingly, humans express two isoforms of Ffar4, short and long (Ffar4S, Ffar4L), differentiated by a 16 amino acid insertion in the third intracellular loop of the long-isoform, whereas other species express only one isoform, homologous to Ffar4S in humans (20). The Ffar4L isoform only signals through $\beta$ Arr2 and is unable to activate $\mathrm{G}_{\mathrm{q} / 11}$-mediated signaling (20). 
Currently, nothing is known about Ffar4 function in the heart. Previous studies have indicated that $\omega 3$-polyunsaturated fatty acids ( $\omega 3$-PUFAs) signaling through Ffar4 attenuate inflammation and obesity (10). Clinical studies indicate that $\omega 3$-PUFAs, eicosapentaenoic acid (EPA) and docosahexaenoic acid (DHA), improve outcomes in coronary heart disease (21-26) and HF (27-30), but the mechanisms underlying this benefit remain unclear. In mice, we were the first to demonstrate that EPA prevents fibrosis and contractile dysfunction in response to pathologic stress in the heart, but EPA was not incorporated into cardiac myocytes or fibroblasts, the traditional mechanism of action for EPA $(16,31)$. However, we found that Ffar4 is expressed in cardiac myocytes and fibroblasts, and that in cardiac fibroblasts, Ffar4 was sufficient and necessary to prevent TGF $\beta 1$-induced fibrosis (16). These finding suggest that Ffar4 might mediate $\omega 3$-PUFA cardioprotection. However, to date, no studies have directly addressed the function of Ffar4 in the heart, nor any link to $\omega 3$-mediated cardioprotection.

Based on these findings, we hypothesized that Ffar4 is a cardioprotective nutrient sensor for medium and long chain fatty acids that resolves inflammation to maintain cardiac homeostasis. To test this hypothesis, we employed mice with systemic deletion of Ffar4 (Ffar4KO mice) to determine if Ffar4 is necessary for an adaptive response to pathologic pressure overload induced by transverse aortic constriction (TAC). Here, we report that TAC induced more severe remodeling in Ffar4KO mice, identifying, for the first time, an entirely novel cardioprotective role for Ffar4 in the heart. In cardiac myocytes, Ffar4 signaling through the

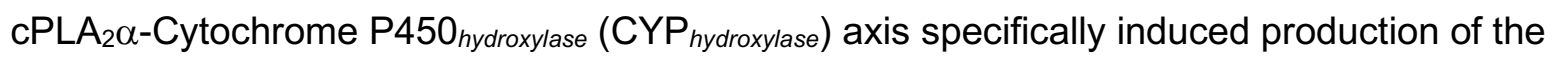
EPA-derived oxylipin 18-hydroxyeicosapentaenoic acid (18-HEPE), the precursor to E-resolvins, a class of inflammation resolving oxylipins. Ffar4-mediated production of 18-HEPE was absent in Ffar4KO cardiac myocytes, providing a potential mechanism to explain the worse remodeling in the Ffar4KO post-TAC. Systemically, loss of Ffar4 altered oxylipin content in circulating plasma lipoproteins characterized by a loss of an anti-inflammatory oxylipins at baseline, and inability to produce both pro-inflammatory and pro-resolving oxylipins following TAC. Finally, we confirmed that Ffar4 is expressed in human heart and down-regulated in heart failure. In summary, our data suggest an entirely novel paradigm whereby FAs function as signaling molecules that activate GPR signaling through Ffar4 to resolve inflammation and maintain cardiac homeostasis. 


\section{Methods}

\section{Mice}

For this study, all experimental mice were placed on a control diet (described below) at 8 weeks of age. At 12 weeks, male and female, WT and Ffar4KO mice were randomized and enrolled into the study. For all experimental analyses, data collection was done with investigator blinded to genotype and treatment.

All procedures on animals conformed to the Public Health Service (PHS) Policy on Humane Care and Use of Laboratory Animals and were reviewed and approved by the Institutional Animal Care and Use Committee at the University of Minnesota.

Ffar4KO mice were generated from cryopreserved sperm from C57BI/6N-

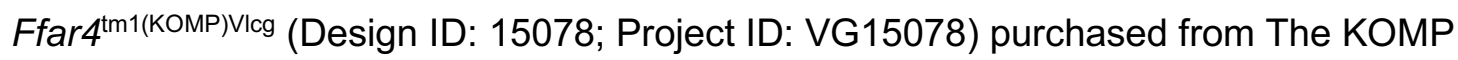
Repository, UC-Davis (Davis, CA, USA). Cryo-recovery of the mouse line was performed at the UMN Mouse Genetics Laboratory through in vitro fertilization (IVF) using C57BI/6J female recipients (\#000664; The Jackson Laboratory, Bar Harbor ME, USA). The line was maintained by hemizygous breeding to $\mathrm{C} 57 \mathrm{BI} / 6 \mathrm{~J}$ for backcrossing. Homozygous mice were crossed to produce wild type and knock out offspring. Genotype was determined by PCR using the primer design provided by KOMP Repository:

Cassette Primers:

Reg-NeoF: GCAGCCTCTGTTCCACATACACTTCA Reg-LacF: ACTTGCTTTAAAAAACCTCCCACA

Gene Specific Primers: $\quad$ Reg-Ffar4-wtR: TGGAAGCCCTCCTCTGTGTTCG Reg-Ffar4-wtF: TTACAGCCAGGTGGGACACAGG

\section{Diet}

Since fatty acids are known agonists of Ffar4, we sought to control the fatty acid profile of the diet by feeding mice a custom chow from Dyets, Inc (\#180539; Bethlehem, PA, USA) beginning at 8 weeks of age. This chow is a modified version of the AIN-93M purified rodent diet used in our previous studies, with corn oil replacing soybean oil (diet composition is listed in Supplemental Table 1) $(16,31)$.

\section{Transverse Aortic Constriction (TAC)}

Transverse aortic constriction (TAC) surgery was performed as previously described (16, 31-34). Baseline measurements of cardiac function by echocardiography were collected prior to surgery. For surgery, mice were anesthetized with $3 \%$ isoflurane and maintained at $1.5 \%$ isoflurane without intubation. A small incision was made slightly left of the midline and above the 
left clavicle without entering the pleural cavity. The muscle tissue and lobes of the thymus were retracted to expose the aortic arch. A 7-0 surgical suture was threaded under the aortic arch and tied-off against a small piece of a blunt 28-gauge needle followed by immediate removal of the needle. To close the original incision, the muscle layer and skin were secured separately with 4-0 continuous sutures. Buprenorphine $(0.1 \mathrm{mg} / \mathrm{kg}$ IP) was administered for pain management during the first 24 hours post-surgery and as needed thereafter. Sham surgery was identical except for ligation of the aorta. Pulsed-wave Doppler by echocardiography was used to confirm pressure gradients by evaluating aortic flow velocity (AoV, Supplemental Tables 3B and 4B) 7-days post-surgery at the site of constriction.

\section{Measurement of cardiac function by echocardiography}

Echocardiography was performed before TAC (baseline), 7-days post-TAC to measure aortic velocity (AoV) to validate the TAC surgery, and 4 weeks post-TAC using the Vevo 2100 (FujiFilm VisualSonics Inc. Toronto, ON, Canada) with a MS550 transducer. For all measurements, mice were anesthetized with isoflurane, gently restrained in the supine position on the prewarmed monitoring pad, and echocardiographic images were captured as mice were recovering from anesthesia to achieve a target heart rate $(\mathrm{HR})$ of $450-500 \mathrm{bpm}$. Parasternal long axis M-mode images of the left ventricle were captured to measure left ventricular wall thicknesses (LVPWs: systolic left ventricular posterior wall; LVPWd: diastolic left ventricular posterior wall), left ventricular internal diameters (LVIDs: systolic left ventricular internal diameter; LVIDd: diastolic left ventricular internal diameter), left ventricular volumes (ESV: end systolic volume, (7.0/(2.4 + LVIDs) ${ }^{\star}$ LVIDs $^{3}$; EDV: end diastolic volume, $(7.0 /(2.4+$ LVIDd))*LVIDd $\left.{ }^{3}\right)$, fractional shortening (FS: 100*((LVIDd - LVIDs)/LVIDd)), ejection fraction (EF: $\left.100^{*}((E D V-E S V) / E D V)\right)$, stroke volume (SV: EDV - ESV), and cardiac output (CO: SV*HR). Pulsed-wave Doppler images of the aortic arch were recorded at the site of constriction to measure peak aortic velocity (AoV) and pressure gradient (PG: $\left.\left(4^{*} \mathrm{AoV}^{2}\right) / 1000\right)$. Pulsed-wave Doppler images of the apical four-chamber view were taken to measure mitral flow velocities (E wave and $A$ wave to calculate $E / A$ ratio) as well as mitral annulus tissue velocity (E/E': peak early transmitral flow velocity/peak early diastolic mitral annular velocity).

\section{Isolation and culture of adult cardiac myocytes}

We previously described procedures for the isolation and culture of adult mouse cardiac myocytes (35). Here, myocytes were isolated 3-days post-TAC from male WT and Ffar4KO, sham and TAC operated mice for analysis of myocyte transcriptomes, or were cultured from WT 
and Ffar4KO hearts for analysis of Ffar4 signaling. Briefly, mice were anesthetized with isoflurane ( $3 \%$ for induction, $1.5 \%$ for maintenance), injected with heparin $(100 \mathrm{IU} / \mathrm{mL})$, the pleural cavity was opened and the heart removed, cannulated on a retrograde perfusion apparatus, and perfused with collagenase type II to dissociate ventricular myocytes. Isolated cardiac myocytes were plated at a density of 50 rod-shaped myocytes per square millimeter on laminin-coated culture dishes. Myocytes were cultured in MEM with Hank's Balanced Salt Solution, $1 \mathrm{mg} / \mathrm{ml}$ bovine serum albumin, $10 \mathrm{mM}$ 2,3-butanedione monoxime, and $100 \mathrm{U} / \mathrm{ml}$ Penicillin in a $4.5 \% \mathrm{CO}_{2}$ incubator $\left(\% \mathrm{CO}_{2}\right.$ determined empirically to maintain culture medium at $\mathrm{pH} 7.0$ ) at $37^{\circ} \mathrm{C}$. All reagents were purchased from Millipore Sigma (Burlington, MA, USA) unless otherwise specified. Full details of the buffers, enzymes, cell culture medium, all procedures for isolation and culturing of adult mouse ventricular myocytes (AMVM) and a detailed diagram of the perfusion apparatus were described in detail previously (35). Based on our previous experience, myocyte isolation are $\sim 95 \%$ pure, but we cannot exclude the possibility of minimal contamination with fibroblasts and endothelial cells (35). Following plating, myocytes were counted at a magnification of $20 \mathrm{X}$ to determine cell viability, and myocytes were incubated in a $4.5 \% \mathrm{CO}_{2}$ incubator at $37^{\circ} \mathrm{C}$ overnight. For analysis of Ffar4 signaling, cardiac myocytes were treated with the Ffar4 agonist TUG-891(Cayman Chemical, MI, USA) (50 $\mu \mathrm{M})$ for 0-60 minutes.

\section{Quantification of erythrocyte FA composition}

At the study endpoint, blood from the posterior vena cava was collected into EDTA tubes. Red blood cell fractions were separated and analyzed for fatty acid composition as previously described $(16,31)$. Briefly, $50 \mu \mathrm{L}$ of isolated erythrocytes were methylated with $14 \%$ boron trifluoride in methanol by incubation for 10 minutes at $100^{\circ} \mathrm{C}$. Fatty acid methyl esters were extracted in hexane and analyzed by a GC-2010 gas chromatography system fitted with a QP2010 mass spectrometer (Shimadzu, Japan) using a Supelco SP-2560 fused silica column (Supelco, Bellefonte, PA). Area counts were obtained using Shimadzu GCMSolution software with multiple ion counts of characteristic fatty acids ions. Each fatty acid was quantified as mass percent of total fatty acids. In general, only minor differences in red blood cell fatty acid composition were detected between WT and Ffar4KO following TAC (Supplemental Table 2).

\section{Tissue histology}

Four weeks after TAC surgery, hearts were arrested in diastole with $60 \mathrm{mM} \mathrm{KCl}$, excised, and weighed. Extracted hearts were cannulated and perfused with PBS with $60 \mathrm{mM} \mathrm{KCl}$, 
followed by $4 \%$ paraformaldehyde. The atria were removed from the fixed hearts prior to embedding in paraffin. Sectioning was performed by AML Laboratories (Jacksonville, FL) providing a transverse view of the ventricles. Lungs were also collected and lung weights were recorded.

Paraffin embedded ventricular sections were deparaffinized with xylene and rehydrated in ethanol. Sections were stained in $0.1 \%$ solution of Sirius red (direct red 80 , Sigma-Aldrich, St Louis, MO) and fast green (Sigma-Aldrich) in 1.2\% picric acid (Ricca Chemical Company, Arlington, TX), followed by dehydration in ethanol and xylene. Sections were imaged at $4 x$ magnification. Ventricular fibrosis (as percent of total ventricular area) was quantified using Fiji software $(\mathrm{NIH})(16,31)$. Ventricular fibrosis was quantified using images captured at $4 \mathrm{X}$ magnification and included both the right and left ventricle. The threshold settings were adjusted to highlight and calculate the total tissue area or picrosirius red positively stained area.

\section{Oxylipin analysis}

Plasma lipoproteins were separated by FPLC followed by measurement of esterified oxylipin (HDL, LDL, and VLDL) or unesterified (albumin). $150 \mu \mathrm{L}$ of mouse EDTA plasma was thawed and filtered by centrifugation at $10,000 \mathrm{~g}$ for $5 \mathrm{~min}$ using Ultrafree Durapore PVDF filter (pore-size $0.2 \mu \mathrm{M}$; Millipore, Bedford, MA). The filtrate was then injected onto an AKTA Purifier FPLC (Amersham Biosciences, Sweden) and run at a flow rate of $0.5 \mathrm{~mL} / \mathrm{min}$ in $1 \mathrm{mM}$ EDTA, $0.9 \% \mathrm{NaCl}$ saline solution, $\mathrm{pH} 7.4$ using an additional $100 \mu \mathrm{L}$ of phosphate buffer solution to fill injection loop. Elutions were monitored at a UV absorbance of $280 \mathrm{~nm}$ and lipoproteins were separated using a Superose-6 10/300 GL size exclusion column. Fractions were collected every 0.5 min using a Foxy 200 fraction collector and were pooled for each lipoprotein fraction and stored at $-80^{\circ} \mathrm{C}$. VLDL, LDL, HDL, and albumin fractions $(100 \mu \mathrm{L})$ were spiked with BHT/EDTA $(0.2 \mathrm{mg} / \mathrm{mL})$, four deuterated octadecanoid and eicosanoid surrogates ( $20 \mu \mathrm{L}$ of $1000 \mathrm{nM}$ concentration with final concentration of $50 \mathrm{nM}$ after reconstitution) and subjected to liquid-liquid extraction to isolate lipid content. Samples were then hydrolyzed in $0.1 \mathrm{M}$ methanolic sodium hydroxide to release ester-linked oxylipins and subjected to solid phase extraction using Chromabond HLB sorbent columns. Oxylipins were eluted with $0.5 \mathrm{~mL}$ of methanol with $0.1 \%$ acetic acid and $1 \mathrm{~mL}$ of ethyl acetate and dried under nitrogen stream and reconstituted in 200 $\mu \mathrm{L}$ methanol acetonitrile (1:1) with $100 \mathrm{nM}$ of 1-cyclohexyluriedo-3-dodecanoic acid used as internal standard.

Samples were analyzed by liquid chromatography using a Waters Acquity UPLC coupled to Waters Xevo triple quadrupole mass spectrometer equipped with electrospray 
ionization source (Waters, Milford, MA). $5 \mu \mathrm{L}$ of the extract was injected and separation was performed using a CORTECS UPLC C18 2.1 x $100 \mathrm{~mm}$ with $1.6 \mu \mathrm{M}$ particle size column. Flow rate was set at $500 \mu \mathrm{L} / \mathrm{min}$ and consisted of a gradient run using water with $0.1 \%$ acetic acid (Solvent A) and acetonitrile isopropanol, 90:10 (Solvent B) for 15 minutes (0-12 min from 25\% B to $95 \% \mathrm{~B}, 12-12.5 \mathrm{~min} 95 \% \mathrm{~B}, 12.5-15 \mathrm{~min} 25 \% \mathrm{~B})$. Electrospray ionization operated in negative ion mode with capillary set at $2.7 \mathrm{kV}$, desolvation temperature set at $600{ }^{\circ} \mathrm{C}$, and source temp set to $150^{\circ} \mathrm{C}$. Optimal oxylipin MRM transitions were identified by direct injection of pure standards onto the mass spectrometer and using cone voltage and collision energy ramps to optimize detection and most prevalent daughter fragments. Calibration curves were generated prior to each run using standards for each oxylipin. Peak detection and integrations were achieved through Target Lynx (Waters, Milford, MA) and each peak inspected for accuracy and corrected when needed.

\section{RNA-seq:}

Three days after surgery, cardiac myocytes were isolated from WT sham $(n=4)$, WT TAC $(n=5)$, Ffar4KO sham $(n=4)$ and Ffar4KO TAC $(n=8)$ male mice. RNA was isolated using RNeasy Fibrous Tissue Mini Kit (Qiagen). Dual-indexed Clontech Pico Mammalian stranded RNA libraries were made. 125bp paired end sequencing was performed using the HiSeq 2500 sequencer (Illumina) by the University of Minnesota Genomics Center.

Data was analyzed by the University of Minnesota Informatics Institute. 2 X 125bp FastQ paired end reads ( $n=8.4$ Million average per sample) were trimmed using Trimmomatic ( $v$ 0.33) enabled with the optional "-q" option; 3bp sliding-window trimming from 3' end requiring minimum Q30. Quality control on raw sequence data for each sample was performed with FastQC. Read mapping was performed via Hisat2 (v2.1.0) using the mouse genome (mm10) as reference. Gene quantification was done via Cuffquant for FPKM values and Feature Counts for raw read counts. Differentially expressed genes were identified using the edgeR (negative binomial) feature in CLC Genomics WorkBench (CLCGWB) (Qiagen, Redwood City, CA) using raw read counts. We filtered the generated list based on a minimum 1.7X Absolute Fold Change and FDR corrected $p<0.05$. Two lists were generated of differentially expressed genes from 1.) WT sham compared to WT TAC animals (2789 genes) and 2.) Ffar4KO sham compared to Ffar4KO TAC animals (1656 genes). The lists were compared to each other to identify genes that overlapped between the two lists to identify common genes (1409) and those genes that were unique to the WT mice (1380 genes) and Ffar4KO mice (247 genes). The genes were 
further annotated in CLCGW based on Gene Ontology (GO) terms for biological function from MGI. Genes were further sorted based on the indicated GO Terms into eight categories.

Principal component analyses used CPMs that were filtered based on gene size (excluding genes less than 200bp) and variance less than 1 in raw read counts. The actual cpm values are log2 transformed and plotted using the PCA function in R.

\section{RT-PCR for Ffar4 and Ffar1 expression in heart}

Human heart samples: Ventricular myocardium was obtained from non-failing and failing human hearts through the Duke Human Heart Repository (DHHR) as previously described (36). Briefly, failing human myocardium was acquired from the left ventricular free wall of explanted hearts following cardiac transplantation. Non-failing left ventricular tissue was acquired from donors whose hearts were not utilized for transplant with permission from Carolina Donor Services. No HIPAA information was provided with any of the samples used in this study. Approximately 20$40 \mathrm{mg}$ of human ventricular myocardium was homogenized in $1 \mathrm{~mL}$ of Trizol (Life Technologies \#15596-026, Carlsbad, CA) with a TissueLyser LT (Qiagen N.V. \#69980, Venlo, The Netherlands). The lysate centrifuged at $12,000 \mathrm{~g}\left(15 \mathrm{~min}\right.$ at $\left.4^{\circ} \mathrm{C}\right)$ after the addition of chloroform $(200 \mu \mathrm{L})$. Isopropanol $(0.5 \mathrm{~mL})$ was then added to the aqueous phase, centrifuged at $12,000 \mathrm{~g}$ (10 $\mathrm{min}$ at $4^{\circ} \mathrm{C}$ ). The resulting RNA pellet was washed with $1 \mathrm{~mL}$ of $75 \%$ ethanol, then centrifuged at $7500 \mathrm{~g}\left(5 \mathrm{~min}\right.$ at $\left.4^{\circ} \mathrm{C}\right)$ and resuspended in RNase-free water.

Primers used to detect human Ffar4:

hFfar4: gctcatctggggctattcg

hFfar4: gcssstcgaaatttcctggt

(The above primers detected Ffar4S (NM_001195755.1) and Ffar4L (NM_181745.3).) hFfar4 aagagctgtcgtgactcacagt (unique for Ffar4L, position 720 - 741) hFfar4 aagagggtgcggaagagc

Note: Using these primers, PCR reactions will detect Ffar4S+Ffar4L or only Ffar4L, but not Ffar4S alone.

\section{Statistics}

Cardiac phenotyping was analyzed using an independent samples, Welch's t-test, comparing WT TAC mice to Ffar4KO TAC mice, to test the hypothesis that the Ffar4KO would have different cardiac phenotypes when under TAC, versus the WT, and to account for the unequal variances observed between the sham and TAC groups. Where specified, principal components analysis (PCA) was used for dimension reduction of oxylipin matrices on log-transformed, 
standardized concentrations. Mixed models were used to account for within mouse timedependent changes in oxylipins (Figure 4) or within mouse lipoprotein differences (Figure 5). Statistical significance is indicated as a $p$ value less than 0.05 ; Tukey's test was used to test for specified post-hoc differences using JMP version 13.2.1. 


\section{Results}

Ffar4 is necessary to mitigate the pathologic hypertrophic response induced by TAC

To test the hypothesis that Ffar4 is cardioprotective, wild-type (WT) and Ffar4KO mice were subjected to TAC, a model of pathologic pressure overload. After four weeks, TAC induced an exaggerated hypertrophic response in male Ffar4KO mice relative to WT, indicated by an increase in heart weight (HW) and heart weight-to-body weight ratio (HW/BW), with no significant difference in BW (Figures $1 \mathrm{~A}$ and B and Supplemental Table 3A). Based on our prior studies demonstrating that in cultured fibroblasts, Ffar4 is sufficient and necessary to prevent TGF $\beta 1$-induced fibrosis, we expected that TAC would produce more fibrosis in the Ffar4KO. However, we were surprised to find that TAC produced similar levels of fibrosis in WT and Ffar4KO hearts (Figures $1 \mathrm{C}$ and D). TAC also caused a trend towards increased lung weights (LW) in male Ffar4KO mice, which is consistent with a worsened response to TAC (Figure 1E). Unexpectedly, the exaggerated hypertrophic response observed in male Ffar4KO mice, was not observed in female Ffar4KO mice. In female mice, TAC induced a similar degree of hypertrophy in WT and Ffar4KO mice, with no significant differences detected in HW, fibrosis, or LW (Figures 1F-H, Supplemental Table 4A).

\section{Ffar4 is necessary to attenuate systolic and diastolic dysfunction induced by TAC}

After four weeks, TAC induced more significant systolic and diastolic dysfunction in male Ffar4KO mice relative to WT, indicated by a more dramatic decrease in ejection fraction (EF, Figure 2A) and a greater increase in E/A ratio (Figure 2B). TAC had only a minor effect on ventricular geometry, with only a trend towards increased end-diastolic and end-systolic volumes (EDV, ESV) (Figures 2C-D, Supplemental Table 3B). However, these results do suggest that male Ffar4KO mice might be progressing to greater eccentric remodeling. In female Ffar4KO and WT hearts, TAC induced a similar degree of systolic and diastolic dysfunction. However, EDV and ESV were both significantly increased, the only significant effects observed in female Ffar4KO mice (Figures 2E-H).

In summary, our results demonstrated that TAC induced a more severe remodeling response in male Ffar4KO mice, indicating for the first time that Ffar4 is necessary for an adaptive response to pathologic stress in the heart. The surprising lack of an exacerbated fibrotic response in the Ffar4KO, as we previously predicted, would indicate that the more severe remodeling observed in the Ffar4KO heart was most likely due to the lack of cardioprotective Ffar4 signaling in cardiac myocytes. Our results also indicated the potential for a significant sex-based difference in the requirement for Ffar4 in response to pathologic stress 
in the heart. There are two potential explanations for this sex-based difference; 1.) The response to TAC in female WT mice was greater than male WT mice $(58 \%$ increase in HW, TAC versus Sham in females, $38 \%$ increase in males, despite similar pressure gradients, Figures $1 \mathrm{~A}$ versus $1 \mathrm{~F}$, and Supplemental Table 3A-B and 4A-B). Given that the response to TAC is time-dependent, perhaps we would see more of a difference if female mice were examined at an earlier time point (or later); or 2.) This represents a significant sex-based difference in Ffar4 function in the heart.

\section{Ffar4 is necessary for induction of cardioprotective inflammatory and cell death genes, and specifically expression of genes associated with phospholipase A2 signaling}

To the best of our knowledge, there are no prior reports regarding the function of Ffar4 in cardiac myocytes. Therefore, to gain insight into the potentially cardioprotective role of Ffar4 in cardiac myocytes, we used RNAseq to analyze transcriptomes of myocytes isolated from WT and Ffar4KO hearts three days post-TAC. Initially, a principal component analysis (PCA) revealed only minor differences in the transcriptomes between genotypes, whereas much larger differences were induced by surgery (Figure $3 \mathrm{~A}$ ). However, a separate analysis of genes specifically regulated by TAC in either WT or Ffar4KO cardiac myocytes identified substantial differences in the number of genes differentially expressed post-TAC depending on genotype (Figure 3B). TAC altered expression of 2,789 genes (up or down) by 1.7-fold or more in WT cardiac myocytes, whereas TAC changed expression of only 1,656 genes in Ffar4KO myocytes. Comparison of these lists revealed 1409 genes were common between the two genotypes, whereas 1380 genes were unique to WT cardiac myocytes, but only 247 genes were unique to Ffar4KO myocytes. (Figure 3B). Individual genes from each genotype were further sorted by Gene Ontology terms for biological function for eight different categories that reflect cardiac myocyte biology; cell death, inflammation, contractile function, angiogenesis, fibrosis, GPR, fatty acid metabolism, and hypertrophy (Figure 3C, Supplemental Tables 5-12). Genes categorized by biological function for cell death and inflammation had the most differentially expressed genes, with significantly more genes identified in WT cardiac myocytes (Figure 3C, Table 1). Interestingly, we also identified genes specifically associated with activation of cPLA $A_{2} \alpha$ signaling, an Ffar4 signaling pathway in macrophages (37), were induced in WT, but not Ffar4KO cardiac myocytes (Figure 3D, Table 1). In summary, transcriptome analysis three days post-TAC suggested that cardiac myocytes from Ffar4KO hearts were unable to induce the same inflammatory and cell death responses as WT animals, specifically genes associated with $\mathrm{CPLA}_{2} \alpha$ signaling, likely contributing to the more severe remodeling in the Ffar4KO post-TAC. 


\section{Ffar4 agonist TUG-891 increases production of 18-HEPE in adult cardiac myocytes}

To define the function of Ffar4 in cardiac myocytes, we focused on Ffar4 mediated activation of $\mathrm{CPLA}_{2} \alpha$, based on the results from our transcriptome analysis. Upon activation, $\mathrm{CPLA}_{2} \alpha$ can translocate to the nuclear membrane and cleave PUFAs from the sn2-acyl bond in membrane phospholipids, traditionally arachidonic acid (AA), but also EPA, DHA, or other PUFAs (38). Cleaved FAs are subsequently metabolized to produce oxylipin products with proinflammatory, anti-inflammatory, or pro-resolving effects. In adult cardiac myocytes treated with the Ffar4 agonist TUG-891, we used UPLC/MS/MS to detect specific AA, EPA, and DHA derived oxylipin production to assess $\mathrm{CPLA}_{2} \alpha$ activity (Figure $4 \mathrm{~A}-\mathrm{H}$ ). Here, oxylipins produced following TUG891 treatment were considered to have one of four fates: 1) acylation into cellular membranes (esterified, myocyte); 2) free in the myocyte (unesterified, myocyte); 3) export in esterified lipids (esterified, medium); or 4) exported as a free oxylipin (unesterified, medium) (Figure 4I). We identified 57 different oxylipins in our samples, with details for MRM transitions, retention times, detection parameters, and detection limits presented in Supplemental Table 13. Results for all oxylipins at 60 minutes are reported in Supplemental Table 14. Surprisingly, we found that TUG-891 selectively increased the production of the EPA-derived oxylipin 18-HEPE, likely by means of $\mathrm{CYP}_{\text {hydrox }}$ activity in cardiac myocytes (esterified 18-HEPE, Figure 4A and unesterified $4 \mathrm{~B}$ ) and exported from myocytes (esterified 18-HEPE, Figure 4C and unesterified 4D), over time (Figures 4E-H).

The Ffar4-depedent increase in 18-HEPE occurred despite AA abundance being much higher, suggesting a previously unrecognized specificity for $\mathrm{CPLA}_{2} \alpha$ function. One explanation for the specific increase in 18-HEPE production could be a relative enrichment of EPA at the nuclear membrane. Because cPLA $2 \alpha$ can translocate to the nuclear membrane (NM) upon activation (Figure 4I), we compared the relative substrate availability in the NM to the sarcolemma/plasma membrane (PM) to determine how this affect the relative availability of PUFAs to oxylipin producing enzymes. \%EPA was lower than \%DHA or \%AA in both PM and NM fractions, however the relative abundance of EPA was 11.5-fold greater in NM compared to PM (Figure 4J). In contrast, \%DHA and \%AA were lower in NM (2.3 and 1.6 fold less, respectively) compared to $P M$, for a total increase in the relative availability of EPA to $C P L A_{2} \alpha$ of approximately 26 -fold relative to $\mathrm{DHA}$ and 19 -fold relative to $\mathrm{AA}$. 


\section{Loss of Ffar4 changes circulating oxylipin content consistent with a loss of anti- inflammatory oxylipins at baseline, and prevents initiation of both mediators of inflammation and of resolution following TAC.}

AA, EPA, and DHA oxylipins are produced intracellularly but are exported and trafficked in plasma in lipoproteins $(39,40)$. Therefore, we examined the consequences of disruption of Ffar4 signaling systemically on circulating oxylipins. Again, we identified 57 different oxylipins in the four plasma fractions, albumin (unesterified), HDL, LDL, and VLDL. To assess global changes in oxylipin phenotypes, we performed PCA comparing the oxylipin content in each fraction for WT and Ffar4KO, sham versus TAC and plot scores for PCA1 and PCA2 for each fraction (Figures 5A-D respectively). PCA loadings, applicable to all plots (Figure 5E), are shown with an example of the loading location of a pro-inflammatory oxylipin (12-HETE), an anti-inflammatory oxylipin (14(15)-EpETE), and a pro-resolving oxylipin (18-HEPE). Hence, a lipoprotein fraction with abundant anti-inflammatory oxylipins would load strictly rightward, a lipoprotein fraction with abundant pro-inflammatory and pro-resolving oxylipins would load downward, and hence an animal with both would load downward and to the right. Detailed loading information for all 57 oxylipins can be found in Supplemental Table 15; tests across all relevant PC scores are given in Supplemental Table 16, and tests across all individual oxylipins are given in Supplemental Table 17.

All albumin fractions were located leftward, or negatively, away from all oxylipins indicating the low concentration of unesterified, freely circulating, oxylipins (Figure 5A). A small adaptive response to TAC surgery in Ffar4KO animals was evidenced by a rightward shift from the Ffar4KO sham location to co-localizing with WT sham and WT TAC.

In all lipoproteins, TAC was associated with an adaptive response in WT mice. For HDL, the oxylipin content of HDL from WT sham mice were located rightward towards 14(15)-EpETE, reflecting high levels of anti-inflammatory EPA-epoxides (Figure 5B). HDL from WT TAC mice were shifted downward and slightly left of WT sham, indicating a replacement of antiinflammatory 14(15)-EpETE with activators of inflammation such as 12-HETE or 15-HETE, paired with production of inflammation resolving oxylipins, 18-HEPE. In contrast, HDL from Ffar4KO sham and TAC mice were both located less rightward and less downward, indicating less overall oxylipin content, less 14(15)-EpETE, and a minimal response to TAC surgery by production of 12-HETE, 15-HETE, and 18-HEPE. Therefore, in the Ffar4KO, the oxylipin profiles indicated an inability to produce oxylipins, especially anti-inflammatory signals at baseline (sham), and an inability to respond to TAC by production of either pro-inflammatory or proresolving oxylipins. 
In a similar fashion, in WT mice, LDL oxylipin profiles indicated an adaptive response following TAC, but not in Ffar4KO mice. Relative to HDL, LDL were generally located more leftward, indicating they contained less oxylipins than HDL, consistent with our prior observations in humans $(39,41)$. LDL from WT sham mice (Figure 5C) were located rightward towards 14(15)-EpETE, while LDL from WT TAC mice moved leftward, away from all oxylipins, consistent with a loss of oxylipin content, co-localizing with LDL from both Ffar4KO sham and TAC mice. This suggested that the loss of Ffar4 pre-disposed towards conditions that were only present in the WT mouse following surgery.

Like LDL, VLDL were more centrally located than HDL, which is evidence of their lower overall oxylipin content relative to HDL, again consistent with prior observations in humans (39, 41). VLDL from WT sham mice (Figure 5D) were located most rightward, reflecting their high concentrations of anti-inflammatory epoxides such as 14(15)-EpETE. However, their moderately positive location upward reflected low levels of pro-inflammatory and pro-resolving oxylipins such as 12-HETE, 15-HETE, and 18-HEPE. The different location of LDL from WT TAC mice, down and to the left, indicated VLDL with less oxylipins overall, but higher levels of the proinflammatory and pro-resolving oxylipins. As with LDL, VLDL from Ffar4KO mice had low oxylipin regardless of their surgery status and were unable to respond to TAC with elevated proinflammatory/pro-resolving oxylipin content.

18-HEPE response to Ffar4 activation was reflected in plasma oxylipin pools, and most 18-HEPE was found in the HDL fraction (Figure 5F). Interestingly, 18-HEPE was dramatically lower in the Ffar4KO HDL fraction at baseline (sham), and although it increased following TAC, it never reached levels observed in the WT. In both LDL and VLDL fractions, 18-HEPE was lower in the Ffar4KO sham, and following TAC, while 18-HEPE levels decreased in the WT, 18HEPE levels actually increased in the Ffar4KO.

\section{Ffar4 is expressed in the human heart and downregulated in HF, with reciprocal upregulation of Ffar1.}

Similar to mice, nothing is known about Ffar4 in the human heart. Therefore, we used RT-PCR to measure Ffar4 expression in samples from both healthy donors and HF patients. We found that while human hearts do express Ffar4, Ffar4 (Ffar4S + Ffar4L) expression is reduced in failing hearts, and Ffar4L represents less than $10 \%$ of total Ffar4 expression, and was not changed following TAC (Figure 6). Unexpectedly, expression of Ffar1, the only other known GPR for medium- and long-chain fatty acids, was reciprocally increased in failing hearts 
bioRxiv preprint doi: https://doi.org/10.1101/776294; this version posted September 25, 2019. The copyright holder for this preprint (which was not certified by peer review) is the author/funder, who has granted bioRxiv a license to display the preprint in perpetuity. It is made available under aCC-BY-NC-ND 4.0 International license.

relative to healthy controls. This represents a significant difference from the mouse heart, which does not express detectable levels of Ffar1 (16). 


\section{Discussion (1438)}

Here, we show for the first time that Ffar4 is cardioprotective and necessary for an adaptive response to pathologic stress in the heart. This is supported by our findings that in Ffar4KO mice, TAC induced an exaggerated hypertrophic response, although with a similar degree of fibrosis, as well as greater systolic and diastolic dysfunction, indicating worse pathologic remodeling. Furthermore, Ffar4-mediated cardioprotection occurred independent of any dietary intervention implying that Ffar4 is able to sense and respond to basal fatty acid composition to protect the heart. Mechanistically, transcriptome analysis of cardiac myocytes three days post-TAC revealed programmatic changes in gene expression associated with inflammation and cell death were lacking in Ffar4KO cardiac myocytes. More importantly, we observed specific deficits in genes associated with $\mathrm{CPLA}_{2} \alpha$-mediated signaling responsible for oxylipin production in Ffar4KO cardiac myocytes. In cultured cardiac myocytes, we were surprised to find that Ffar4 preferentially increased production of one cardioprotective, EPAderived oxylipin, 18-HEPE, despite the generally low levels of EPA detected in cardiac myocyte membranes at baseline. Furthermore, profiling oxylipin content in plasma, both esterified in circulating lipoproteins and unesterified (bound to albumin), generally indicated that loss of Ffar4 was associated with lower overall oxylipin content in all plasma fractions, and specifically less anti-inflammatory oxylipins at baseline, and inability to increase pro-inflammatory and proresolving oxylipins following TAC. Together, these results suggest that a key mechanism to explain the cardioprotective effects of Ffar4 is through induction of pro-resolving oxylipins in cardiac myocytes directly, and through indirect effects in balancing pro-inflammatory and proresolving oxylipin composition systemically, consistent with failure to activate $c P L A_{2} \alpha$.

Additionally, we demonstrated that Ffar4 is expressed in the human heart, and downregulated in HF. In summary, our data suggest that Ffar4 functions as a nutrient sensor for medium and long chain fatty acids to resolve inflammation and maintain cardiac homeostasis.

Transcriptome analysis of cardiac myocytes performed three days post-TAC identified a deficit in $\mathrm{CPLA}_{2} \alpha$ signaling in Ffar4 cardiac myocytes (Figure 3) (38). There are six cPLA $\mathrm{A}_{2}$ family members $(\alpha, \beta, \gamma, \delta, \varepsilon$, and $\zeta)$, which show only roughly $30 \%$ sequence homology, but have different enzymatic properties, tissue expression, and subcellular localizations (38). cPLA2 $\alpha$ is widely expressed and has biologic functions in a multitude of cell types (38) and upon activation, it localizes to the nuclear membrane (42). Early studies suggested that $\mathrm{CPLA}_{2} \alpha$ functions as a negative regulator of striated muscle growth. In mice with systemic deletion of $\mathrm{cPLA} 2 \alpha$ (cPLA ${ }_{2} \mathrm{KO}$ mice), normal developmental growth of skeletal and cardiac muscle, as well as 
pathologic cardiac hypertrophic growth post-TAC are exaggerated (43). Mechanistically, loss of $\mathrm{cPLA}_{2} \alpha$ induced sustained activation of IGF-1 signaling, a known hypertrophic stimulus, in cardiac myocytes from $\mathrm{cPLA}{ }_{2} \mathrm{KO}$ hearts (43). In addition, $\mathrm{cPLA}_{2} \alpha$ might protect against ischemic injury in the heart, although there are conflicting reports regarding $\mathrm{CPLA}_{2} \alpha$-mediated cardioprotection $(44,45)$. Our results demonstrate that $\mathrm{CPLA}_{2} \alpha(P l a 2 g 4 a)$ was upregulated 2.8 fold only in WT cardiac myocytes, whereas IGF-1 (Igf1) was upregulated 2.8 fold only in Ffar4KO cardiac myocytes, consistent with the exaggerated hypertrophic response post-TAC in hearts from Ffar4KO mice. Furthermore, the deficit in $\mathrm{CPLA}_{2} \alpha$ mediated signaling in Ffar4KO cardiac myocytes was associated with worse remodeling following TAC, suggesting Ffar4$\mathrm{CPLA}_{2} \alpha$ signaling is cardioprotective.

Functionally, cPLA $2 \alpha$ (Pla2g4a) cleaves PUFAs from the sn2-acyl bond in membrane phospholipids. AA is the most common substrate, but DHA and EPA are substrates, leading to the production of downstream oxylipins (38). Initially, cleaved FAs (AA, EPA, DHA) are metabolized by cyclooxygenases (COX1/2), lipoxygenases (5-LOX, 12/15-LOX), or CYP hydroxylase $_{\text {. }}$ and $\mathrm{CYP}_{\text {epoxygenase }}$ to generate oxygenated signaling mediators, including leukotrienes and prostaglandins, which are collectively known as oxylipins, many of which mediate pro- or antiinflammatory responses, or initiate resolution of inflammation (46). Here, we found that in cardiac myocytes, the Ffar4 agonist TUG-891 activated $c P L A_{2} \alpha$ and induced the production of a specific EPA-derived oxylipin, 18-HEPE (Figure 4, Supplemental Tables 14). Although, the

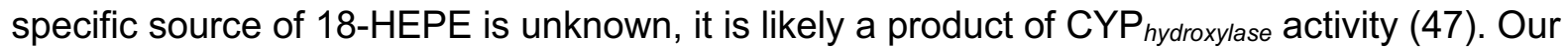
data imply a role for Cyp26b1 which is previously identified for its role in hydroxylating retinoic acid (48). While this is the first such demonstration in cardiac myocytes, one previous study in macrophages indicated that DHA signaling through Ffar4 activates $\mathrm{CPLA}_{2} \alpha$ mediated oxylipin production (37). Interestingly, we found EPA levels in red blood cells were low, $0.1-0.2 \%$ of total membrane FAs, whereas DHA was $4 \%$ and AA was $13 \%$ (Supplemental Tables $2 \mathrm{~A}$ and $2 \mathrm{~B}$ ), similar to our previous studies (16). Furthermore, we previously found that in cardiac myocytes, EPA was $0.5 \%$ of total membrane FAs, whereas DHA was $10 \%$ and AA was about $12 \%$ (16). Therefore, in cardiac myocytes, Ffar4 shows surprising degree of specificity for the production of 18-HEPE, largely to the exclusion of other EPA, DHA, or AA derived oxylipins despite the low levels of EPA in cardiac myocyte membranes. Potentially, the localization of cPLA $2 \alpha$ to an EPA rich membrane enhances its capacity to mediate the specific production of EPA-derived oxylipins such as 18-HEPE. 
While there are no previous studies regarding the direct effects of 18-HEPE in cardiac myocytes, macrophages from fat-1 mice, which have high endogenous levels of $\omega 3-P U F A s$, particularly EPA, due to overexpression the c. elegans fat-1 gene, produced high levels of 18HEPE that were associated with cardioprotection in fat-1 mice post-TAC (49). Furthermore, direct i.p. injection of 18-HEPE attenuated remodeling post-TAC (49). Interestingly, 18-HEPE is also the precursor for E-series resolvins (RvE1, RvE2, and RvE3), a class of pro-resolving oxylipins (50). E-resolvins signal through ERV1/ChemR23, an orphan GPR that binds to and is activated by both E-resolvins and the endogenous peptide chemerin, suggesting a potential mechanism to explain EPA-mediated inflammation resolving effects (51). ERV1/ChemR23 is expressed in the heart, and preconditioning with RvE1 reduces ischemia/reperfusion injury (52), while RvE1 infusion one-week following coronary artery ligation attenuates post-MI inflammatory response (53). In our study, the production of 18-HEPE co-occurred systemically with the production of pro-inflammatory products, 12-HETE and 15-HETE. Combined with our data, these studies suggest that Ffar4-cPLA $2 \alpha$ mediated production of 18-HEPE is cardioprotective by providing the capacity to resolve inflammation, either through direct effects in the heart, released from infiltrating macrophages or directly produced in cardiac myocytes, or indirect effects via production of E-resolvins and activation of ERV1/ChemR23.

Two important deficits were observed in circulating oxylipin profiles in Ffar4KO mice: first, Ffar4KO mice lacked the capacity to induce any cPLA ${ }_{2} \alpha$ mediated oxylipin response, and second, they lacked the capacity to modify oxylipin production in response to TAC. Surprisingly, this did not mean suppressing the activation of inflammation, but instead it meant co-producing mediators essential for the resolution of inflammation. Systemically, WT mice at baseline (sham) had the highest oxylipin content, with high levels of anti-inflammatory oxylipins such as 14(15)EpETE. Interventions that increase the production of EpETEs, or slow their inactivation, attenuate TAC remodeling $(54,55)$, but we observed that TAC altered the HDL oxylipin content to include a pro-inflammatory response (12-HETE, 15-HETE). In mice, 12/15-LOX is the common source for both 12- and 15-HETE, and overexpression of 12/15-LOX is associated with increased levels of unesterified 12-HETE, 15-HETE, and heart failure (56). We found that despite increase in this pro-inflammatory insult (increased 12-HETE, 15-HETE), WT mice were protected through their capacity to co-produce the pro-resolving 18-HEPE, which was lacking in Ffar4KO mice. This suggests that the capacity to produce 12/15-LOX metabolites is beneficial, provided they can also initiate recovery.

Clinically, $\omega 3$-PUFAs are important to cardiovascular health $(57,58)$, and there is substantial evidence demonstrating a benefit of $\omega 3$-PUFAs in coronary heart disease (CHD) and 
HF. In one large clinical trial, Gruppo Italiano per lo Studio della Sopravvivenza nell'Infarto miocardico-Heart Failure (GISSI-HF) (27), and several smaller trials, $\omega 3-P U F A s$ improved HF outcomes (28-30). In animal models, we have also demonstrated that EPA, in a concentrationdependent manner, prevents pathologic remodeling post-TAC $(16,31,59)$. However, important questions remain regarding the mechanism underlying the benefit of $\omega 3$-supplementation in HF. Here, we found that Ffar4, a receptor for medium and long-chain fatty acids including, but not limited to, $\omega 3$-PUFAs, is cardioprotective, but whether Ffar4 is required for $\omega 3$-mediated protection is unclear at this time. Mechanistically, we found that in cardiac myocytes Ffar4 increased 18-HEPE levels, which might be directly cardioprotective or act through its downstream metabolites E-resolvins. Ultimately, this could suggest a feed-forward mechanism with multiple points for EPA mediated cardioprotection: 1. Through Ffar4-mediated production of 18-HEPE, either in cardiac myocytes (Figure 4) or potentially macrophages (37). 2. Increasing overall EPA levels, thereby increasing 18-HEPE levels, as suggested by others (49), or 3. Increased E-resolvin levels and activation of ERV1/ChemR23 either via Ffar mediated production of 18-HEPE or increased 18-HEPE levels secondary to an overall increase in EPA levels.

In conclusion, our results identify Ffar4 as a nutrient sensor for medium and long chain FAs that attenuates inflammation to maintain cardiac homeostasis. Finally, our findings suggest a novel paradigm for FAs in cardiac myocytes, whereby FA function not simply as an energy source, but as signaling molecules that activate cardioprotective GPR signaling. 


\section{Acknowledgements:}

This work was supported by NIH HL130099 (TDO and GCS), Minnesota Obesity

Prevention Training Program T32 NIH Grant 1T32DK083250-01A1 (KM), and a grant from

Amarin Corporation. The authors also acknowledge the Genomics Core at the University of

Minnesota for technical expertise on all data acquisition and analysis for transcriptome

phenotypes.

\section{Conflict of Interest:}




\section{Abbreviations:}

12-HETE, 12-hydroxyeicosatrienoic acid

14(15)-EpETE, 14(15)-epoxytetraenoic acid

15-HETE, 15-hydroxyeicosatrienoic acid

18-HEPE, 18-hydroxyeicosapentaenoic acid

$A A$, arachidonic acid

AMVM, adult mouse ventricular myocytes

ATM, adipose tissue macrophage

$\beta$ Arr2, $\beta$ arrestin-2

CANTOS, Canakinumab Anti-inflammatory Thrombosis Outcome Study

$\mathrm{cPLA}_{2} \alpha$, cytoplasmic phospholipase $\mathrm{A}_{2} \alpha$

COX, cyclooxygenase

CYP, cytochrome p450

DHA, docosahexaenoic acid

EPA, eicosapentaenoic acid

FA, fatty acid

Ffar, free fatty acid receptor

Ffar1, free fatty acid receptor 1

Ffar4, free fatty acid receptor 4

Ffar4KO, free fatty acid receptor 4 knock out mouse

Ffar4S, free fatty acid receptor 4 short isoform

Ffar4L, free fatty acid receptor 4 long isoform

FPLC, fast performance liquid chromatography

GISSI-HF, Gruppo Italiano per lo Studio della Sopravvivenza nell'Infarto miocardico-Heart

Failure

GO, gene ontology

GPR, G-coupled protein receptor

GPR120, G-protein coupled receptor 120

$\mathrm{HF}$, heart failure

HFrEF, heart failure reduced ejection fraction

$\mathrm{HFpEF}$, heart failure preserved ejection fraction

$\mathrm{HDL}$, high density lipoproteins

$\mathrm{IL}-1 \beta$, interleukin-1 $\beta$

$\mathrm{iPLA}_{2}$, calcium-independent PLA 2

$\mathrm{KO}$, knock outTAC, transverse aortic constriction

KOMP, Knock Out Mouse Project

LDL, low density lipoproteins

LOX, lipoxygenase

MUFA, monounsaturated fatty acid

$\mathrm{NM}$, nuclear membrane

PAF-AH , platelet-activating acetylhydrolases

PCA, principal component analysis

PM, plasma membrane

PUFA, polyunsaturated fatty acid

SFA, saturated fatty acid

TAC, transverse aortic constriction

TGF $\beta-1$; transforming growth factor beta-1WT, wild type

VLDL, very low density lipoproteins

WT, wild type 


\section{References}

1. Prabhu SD, and Frangogiannis NG. The Biological Basis for Cardiac Repair After Myocardial Infarction: From Inflammation to Fibrosis. Circ Res. 2016;119(1):91-112.

2. Lam CSP, Voors AA, de Boer RA, Solomon SD, and van Veldhuisen DJ. Heart failure with preserved ejection fraction: from mechanisms to therapies. Eur Heart $J$. 2018;39(30):2780-92.

3. Paulus WJ, and Tschope C. A novel paradigm for heart failure with preserved ejection fraction: comorbidities drive myocardial dysfunction and remodeling through coronary microvascular endothelial inflammation. J Am Coll Cardiol. 2013;62(4):263-71.

4. Shah SJ, Kitzman DW, Borlaug BA, van Heerebeek L, Zile MR, Kass DA, et al. Phenotype-specific treatment of heart failure with preserved ejection fraction: $A$ multiorgan roadmap. Circulation. 2016;134(1):73-90.

5. Ridker PM, Everett BM, Thuren T, MacFadyen JG, Chang WH, Ballantyne C, et al. Antiinflammatory Therapy with Canakinumab for Atherosclerotic Disease. $N$ Engl $J$ Med. 2017;377(12):1119-31.

6. Rymer JA, and Newby LK. Failure to Launch: Targeting Inflammation in Acute Coronary Syndromes. JACC Basic Trans/ Sci. 2017;2(4):484-97.

7. Alvarez-Curto $\mathrm{E}$, and Milligan $\mathrm{G}$. Metabolism meets immunity: The role of free fatty acid receptors in the immune system. Biochem Pharmacol. 2016;114:3-13.

8. Ichimura A, Hasegawa S, Kasubuchi M, and Kimura I. Free fatty acid receptors as therapeutic targets for the treatment of diabetes. Front Pharmacol. 2014;5:236.

9. Christiansen E, Watterson KR, Stocker CJ, Sokol E, Jenkins L, Simon K, et al. Activity of dietary fatty acids on FFA1 and FFA4 and characterisation of pinolenic acid as a dual FFA1/FFA4 agonist with potential effect against metabolic diseases. Br J Nutr. 2015;113(11):1677-88.

10. Oh DY, Talukdar S, Bae EJ, Imamura T, Morinaga H, Fan W, et al. GPR120 is an omega-3 fatty acid receptor mediating potent anti-inflammatory and insulin-sensitizing effects. Cell. 2010;142(5):687-98.

11. Suckow AT, Polidori D, Yan W, Chon S, Ma JY, Leonard J, et al. Alteration of the glucagon axis in GPR120 (FFAR4) knockout mice: a role for GPR120 in glucagon secretion. J Biol Chem. 2014;289(22):15751-63.

12. Moran BM, Abdel-Wahab YH, Flatt PR, and McKillop AM. Evaluation of the insulinreleasing and glucose-lowering effects of GPR120 activation in pancreatic beta-cells. Diabetes Obes Metab. 2014;16(11):1128-39.

13. Stone VM, Dhayal S, Brocklehurst KJ, Lenaghan C, Sorhede Winzell M, Hammar M, et al. GPR120 (FFAR4) is preferentially expressed in pancreatic delta cells and regulates somatostatin secretion from murine islets of Langerhans. Diabetologia. 2014;57(6):118291.

14. Gotoh $\mathrm{C}$, Hong $\mathrm{YH}$, Iga $\mathrm{T}$, Hishikawa D, Suzuki $\mathrm{Y}$, Song $\mathrm{SH}$, et al. The regulation of adipogenesis through GPR120. Biochem Biophys Res Commun. 2007;354(2):591-7.

15. Quesada-Lopez T, Cereijo R, Turatsinze JV, Planavila A, Cairo M, Gavalda-Navarro A, et al. The lipid sensor GPR120 promotes brown fat activation and FGF21 release from adipocytes. Nat Commun. 2016;7:13479.

16. Eclov JA, Qian Q, Redetzke R, Chen Q, Wu SC, Healy CL, et al. EPA, not DHA, prevents fibrosis in pressure overload induced heart failure; potential role of free fatty acid receptor 4. J Lipid Res. 2015;56:2297-308.

17. Hirasawa A, Tsumaya K, Awaji T, Katsuma S, Adachi T, Yamada M, et al. Free fatty acids regulate gut incretin glucagon-like peptide-1 secretion through GPR120. Nat Med. 2005;11(1):90-4. 
18. Tanaka T, Yano T, Adachi T, Koshimizu TA, Hirasawa A, and Tsujimoto G. Cloning and characterization of the rat free fatty acid receptor GPR120: in vivo effect of the natural ligand on GLP-1 secretion and proliferation of pancreatic beta cells. Naunyn Schmiedebergs Arch Pharmacol. 2008;377(4-6):515-22.

19. Hudson BD, Shimpukade B, Mackenzie AE, Butcher AJ, Pediani JD, Christiansen E, et al. The pharmacology of TUG-891, a potent and selective agonist of the free fatty acid receptor 4 (FFA4/GPR120), demonstrates both potential opportunity and possible challenges to therapeutic agonism. Mol Pharmacol. 2013;84(5):710-25.

20. Watson SJ, Brown AJ, and Holliday ND. Differential signaling by splice variants of the human free fatty acid receptor GPR120. Mol Pharmacol. 2012;81(5):631-42.

21. Dietary supplementation with $n-3$ polyunsaturated fatty acids and vitamin $E$ after myocardial infarction: results of the GISSI-Prevenzione trial. Gruppo Italiano per lo Studio della Sopravvivenza nell'Infarto Miocardico. Lancet. 1999;354(9177):447-55.

22. Macchia A, Levantesi G, Franzosi MG, Geraci E, Maggioni AP, Marfisi R, et al. Left ventricular systolic dysfunction, total mortality, and sudden death in patients with myocardial infarction treated with n-3 polyunsaturated fatty acids. Eur $J$ Heart Fail. 2005;7(5):904-9.

23. Marchioli R, Barzi F, Bomba E, Chieffo C, Di Gregorio D, Di Mascio R, et al. Early protection against sudden death by $n-3$ polyunsaturated fatty acids after myocardial infarction: time-course analysis of the results of the Gruppo Italiano per lo Studio della Sopravvivenza nell'Infarto Miocardico (GISSI)-Prevenzione. Circulation. 2002;105(16):1897-903.

24. Burr ML, Fehily AM, Gilbert JF, Rogers S, Holliday RM, Sweetnam PM, et al. Effects of changes in fat, fish, and fibre intakes on death and myocardial reinfarction: diet and reinfarction trial (DART). Lancet. 1989;2(8666):757-61.

25. Mozaffarian D, Lemaitre RN, King IB, Song X, Spiegelman D, Sacks FM, et al. Circulating long-chain omega-3 fatty acids and incidence of congestive heart failure in older adults: the cardiovascular health study: a cohort study. Ann Intern Med. 2011;155(3):160-70.

26. Yokoyama M, Origasa H, Matsuzaki M, Matsuzawa $Y$, Saito $Y$, Ishikawa $Y$, et al. Effects of eicosapentaenoic acid on major coronary events in hypercholesterolaemic patients (JELIS): a randomised open-label, blinded endpoint analysis. Lancet. 2007;369(9567):1090-8.

27. Tavazzi L, Maggioni AP, Marchioli R, Barlera S, Franzosi MG, Latini R, et al. Effect of n3 polyunsaturated fatty acids in patients with chronic heart failure (the GISSI-HF trial): a randomised, double-blind, placebo-controlled trial. Lancet. 2008;372(9645):1223-30.

28. Nodari S, Triggiani M, Campia U, Manerba A, Milesi G, Cesana BM, et al. Effects of n-3 polyunsaturated fatty acids on left ventricular function and functional capacity in patients with dilated cardiomyopathy. J Am Coll Cardiol. 2011;57(7):870-9.

29. Chrysohoou C, Metallinos G, Georgiopoulos G, Mendrinos D, Papanikolaou A, Magkas $\mathrm{N}$, et al. Short term omega-3 polyunsaturated fatty acid supplementation induces favorable changes in right ventricle function and diastolic filling pressure in patients with chronic heart failure; A randomized clinical trial. Vascul Pharmacol. 2016;79:43-50.

30. Kohashi K, Nakagomi A, Saiki Y, Morisawa T, Kosugi M, Kusama Y, et al. Effects of eicosapentaenoic acid on the levels of inflammatory markers, cardiac function and longterm prognosis in chronic heart failure patients with dyslipidemia. J Atheroscler Thromb. 2014;21(7):712-29.

31. Chen J, Shearer GC, Chen Q, Healy CL, Beyer AJ, Nareddy VB, et al. Omega-3 fatty acids prevent pressure overload-induced cardiac fibrosis through activation of cyclic GMP/protein kinase G signaling in cardiac fibroblasts. Circulation. 2011;123(6):584-93. 
32. O'Connell TD, Ishizaka S, Nakamura A, Swigart PM, Rodrigo MC, Simpson GL, et al. The alpha(1A/C)- and alpha(1B)-adrenergic receptors are required for physiological cardiac hypertrophy in the double-knockout mouse. J Clin Invest. 2003;111(11):1783-91.

33. O'Connell TD, Swigart PM, Rodrigo MC, Ishizaka S, Joho S, Turnbull L, et al. Alpha1adrenergic receptors prevent a maladaptive cardiac response to pressure overload. $J$ Clin Invest. 2006;116(4):1005-15.

34. Nakamura A, Rokosh DG, Paccanaro M, Yee RR, Simpson PC, Grossman W, et al. LV systolic performance improves with development of hypertrophy after transverse aortic constriction in mice. Am J Physiol Heart Circ Physiol. 2001;281(3):H1104-12.

35. O'Connell TD, Rodrigo MC, and Simpson PC. Isolation and culture of adult mouse cardiac myocytes. Methods Mol Biol. 2007;357:271-96.

36. Schechter MA, Hsieh MK, Njoroge LW, Thompson JW, Soderblom EJ, Feger BJ, et al. Phosphoproteomic profiling of human myocardial tissues distinguishes ischemic from non-ischemic end stage heart failure. PLoS One. 2014;9(8):e104157.

37. Liu Y, Chen LY, Sokolowska M, Eberlein M, Alsaaty S, Martinez-Anton A, et al. The fish oil ingredient, docosahexaenoic acid, activates cytosolic phospholipase $A(2)$ via GPR120 receptor to produce prostaglandin $E(2)$ and plays an anti-inflammatory role in macrophages. Immunology. 2014;143(1):81-95.

38. Leslie CC. Cytosolic phospholipase $A(2)$ : physiological function and role in disease. $J$ Lipid Res. 2015;56(8):1386-402.

39. Newman JW, Pedersen TL, Brandenburg VR, Harris WS, and Shearer GC. Effect of omega-3 fatty acid ethyl esters on the oxylipin composition of lipoproteins in hypertriglyceridemic, statin-treated subjects. PLoS One. 2014;9(11):e111471.

40. Shearer GC, and Newman JW. Lipoprotein lipase releases esterified oxylipins from very low-density lipoproteins. Prostaglandins Leukot Essent Fatty Acids. 2008;79(6):215-22.

41. Shearer GC, Borkowski K, Puumala SL, Harris WS, Pedersen TL, and Newman JW. Abnormal lipoprotein oxylipins in metabolic syndrome and partial correction by omega-3 fatty acids. Prostaglandins Leukot Essent Fatty Acids. 2018;128:1-10.

42. Peters-Golden M, Song K, Marshall T, and Brock T. Translocation of cytosolic phospholipase $A 2$ to the nuclear envelope elicits topographically localized phospholipid hydrolysis. Biochem J. 1996;318 ( Pt 3):797-803.

43. Haq S, Kilter H, Michael A, Tao J, O'Leary E, Sun XM, et al. Deletion of cytosolic phospholipase A2 promotes striated muscle growth. Nat Med. 2003;9(7):944-51.

44. Kerkela R, Boucher M, Zaka R, Gao E, Harris D, Piuhola J, et al. Cytosolic phospholipase $A(2)$ alpha protects against ischemia/reperfusion injury in the heart. Clin Transl Sci. 2011;4(4):236-42.

45. Saito Y, Watanabe K, Fujioka D, Nakamura T, Obata JE, Kawabata K, et al. Disruption of group IVA cytosolic phospholipase $A(2)$ attenuates myocardial ischemia-reperfusion injury partly through inhibition of TNF-alpha-mediated pathway. Am J Physiol Heart Circ Physiol. 2012;302(10):H2018-30.

46. Shearer GC, and Walker RE. An overview of the biologic effects of omega- 6 oxylipins in humans. Prostaglandins Leukot Essent Fatty Acids. 2018;137:26-38.

47. Gabbs M, Leng S, Devassy JG, Monirujjaman M, and Aukema HM. Advances in Our Understanding of Oxylipins Derived from Dietary PUFAs. Adv Nutr. 2015;6(5):513-40.

48. White JA, Ramshaw H, Taimi M, Stangle W, Zhang A, Everingham S, et al. Identification of the human cytochrome P450, P450RAl-2, which is predominantly expressed in the adult cerebellum and is responsible for all-trans-retinoic acid metabolism. Proc Natl Acad Sci U S A. 2000;97(12):6403-8.

49. Endo J, Sano M, Isobe Y, Fukuda K, Kang JX, Arai H, et al. 18-HEPE, an n-3 fatty acid metabolite released by macrophages, prevents pressure overload-induced maladaptive cardiac remodeling. J Exp Med. 2014;211(8):1673-87. 
50. Dalli J, and Serhan CN. Identification and structure elucidation of the pro-resolving mediators provides novel leads for resolution pharmacology. $\mathrm{Br} \mathrm{J}$ Pharmacol. 2019;176(8):1024-37.

51. Arita M, Bianchini F, Aliberti J, Sher A, Chiang N, Hong S, et al. Stereochemical assignment, antiinflammatory properties, and receptor for the omega-3 lipid mediator resolvin E1. J Exp Med. 2005;201(5):713-22.

52. Keyes KT, Ye Y, Lin Y, Zhang C, Perez-Polo JR, Gjorstrup P, et al. Resolvin E1 protects the rat heart against reperfusion injury. Am J Physiol Heart Circ Physiol. 2010;299(1):H153-64.

53. Liu G, Liu Q, Shen Y, Kong D, Gong Y, Tao B, et al. Early treatment with Resolvin E1 facilitates myocardial recovery from ischaemia in mice. $\mathrm{Br} \mathrm{J}$ Pharmacol. 2018;175(8):1205-16.

54. Westphal C, Spallek B, Konkel A, Marko L, Qadri F, DeGraff LM, et al. CYP2J2 overexpression protects against arrhythmia susceptibility in cardiac hypertrophy. PLoS One. 2013;8(8):e73490.

55. Xu D, Li N, He Y, Timofeyev V, Lu L, Tsai HJ, et al. Prevention and reversal of cardiac hypertrophy by soluble epoxide hydrolase inhibitors. Proc Natl Acad Sci U S A. 2006;103(49):18733-8.

56. Kayama Y, Minamino T, Toko H, Sakamoto M, Shimizu I, Takahashi H, et al. Cardiac $12 / 15$ lipoxygenase-induced inflammation is involved in heart failure. J Exp Med. 2009;206(7):1565-74.

57. De Caterina R. n-3 fatty acids in cardiovascular disease. N Engl J Med. 2011;364(25):2439-50.

58. Mozaffarian D, and $\mathrm{Wu} \mathrm{JH}$. Omega-3 fatty acids and cardiovascular disease: effects on risk factors, molecular pathways, and clinical events. J Am Coll Cardiol. 2011;58(20):2047-67.

59. O'Connell TD, Block RC, Huang SP, and Shearer GC. omega3-Polyunsaturated fatty acids for heart failure: Effects of dose on efficacy and novel signaling through free fatty acid receptor 4. J Mol Cell Cardiol. 2017;103:74-92.

60. Harouki N, Nicol L, Remy-Jouet I, Henry JP, Dumesnil A, Lejeune A, et al. The IL-1beta Antibody Gevokizumab Limits Cardiac Remodeling and Coronary Dysfunction in Rats With Heart Failure. JACC Basic Trans/ Sci. 2017;2(4):418-30.

61. Van Tassell BW, Toldo S, Mezzaroma E, and Abbate A. Targeting interleukin-1 in heart disease. Circulation. 2013;128(17):1910-23.

62. Otterbein LE, Foresti R, and Motterlini R. Heme Oxygenase-1 and Carbon Monoxide in the Heart: The Balancing Act Between Danger Signaling and Pro-Survival. Circ Res. 2016;118(12):1940-59.

63. Mitchell JA, and Kirkby NS. Eicosanoids, prostacyclin and cyclooxygenase in the cardiovascular system. Br J Pharmacol. 2019;176(8):1038-50. 


\section{Figure 1. Ffar4 is necessary to mitigate the pathologic hypertrophic response induced by TAC.}

A

$\mathrm{E}$

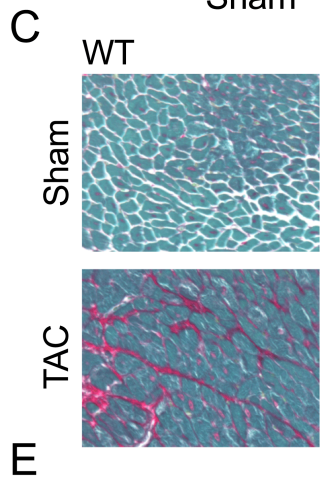

Ffar4 KO

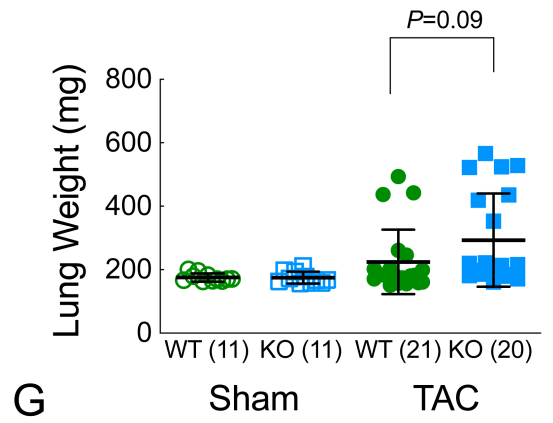

$G$

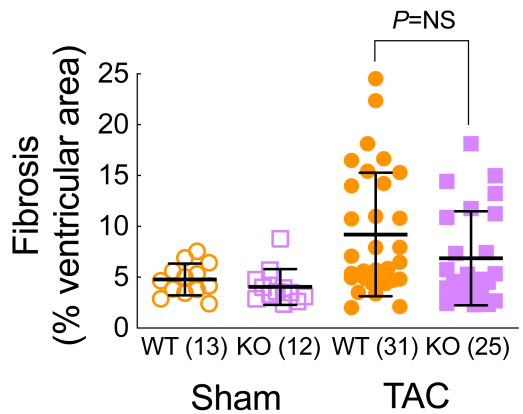

B

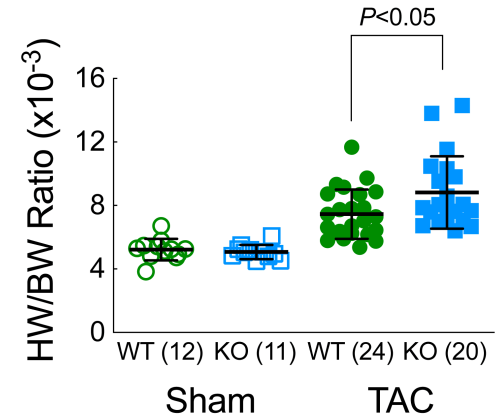

D
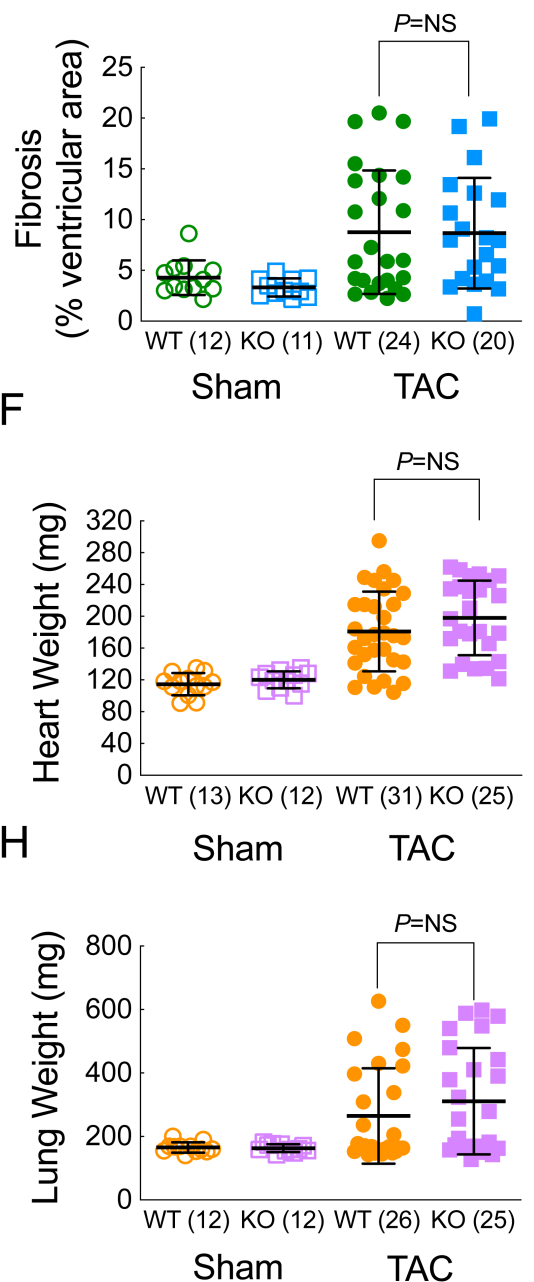

Figure 1. Four weeks following TAC or sham surgery, mice were euthanized and hearts were collected for morphological analysis. A, Heart weight (HW) and B, heart weight-to-body weight ratio (HW/BW) of male WT and Ffar4KO mice. C, Representative images of ventricular fibrosis quantified from ventricular cross sections from male WT and Ffar4KO mice stained with Sirius red to measure fibrosis, with Fast Green used as a counterstain. D, Ventricular fibrosis quantified by fibrotic area (Sirius red)/total ventricular area (Fast green) from male WT and Ffar4KO mice. E, Lung weight of male WT and Ffar4KO mice. F, Heart weight; $\mathbf{G}$, fibrosis and $\mathbf{H}$, lung weight for female WT and Ffar4KO mice. Data were compared by a Welch's two sample t test. Error bars represent the mean with SD. 
Figure 2. Ffar4 is necessary to attenuate systolic and diastolic dysfunction induced by TAC.

A

C
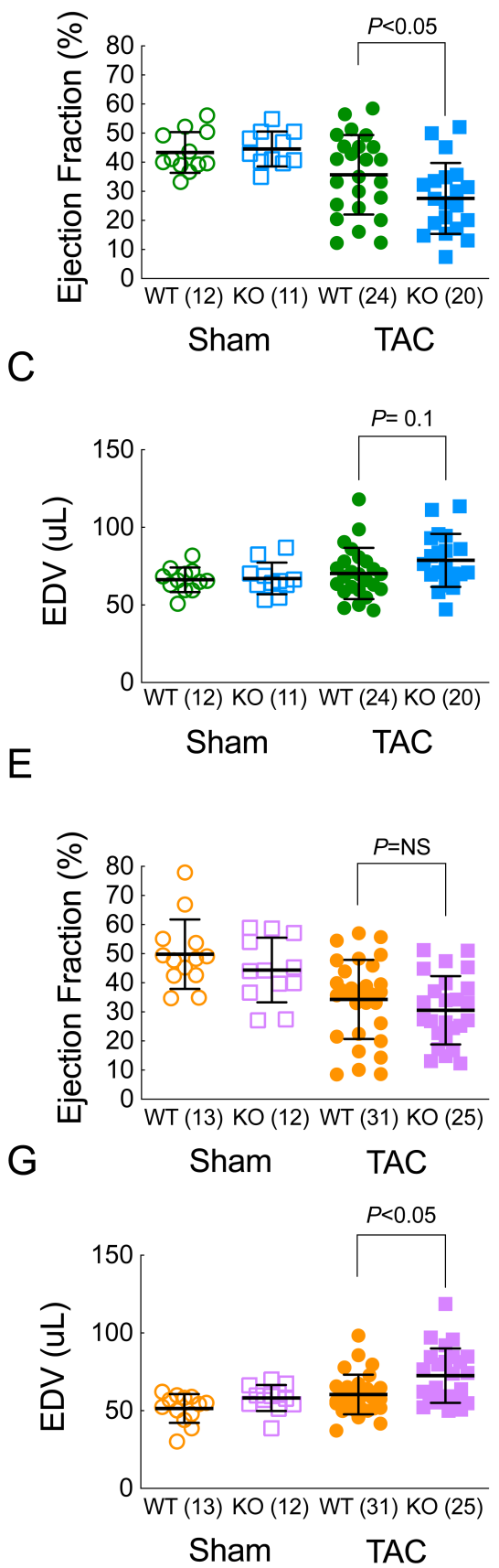

B

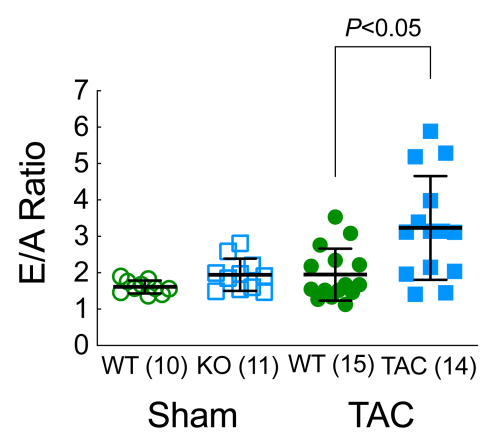

D

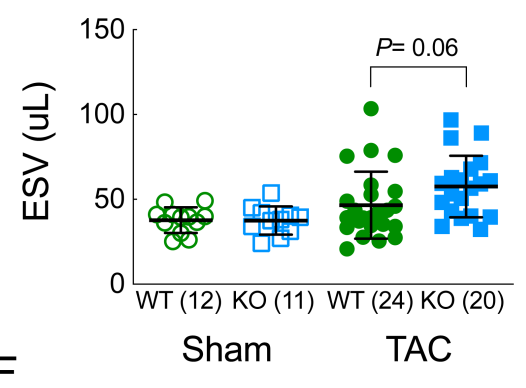

$\mathrm{F}$
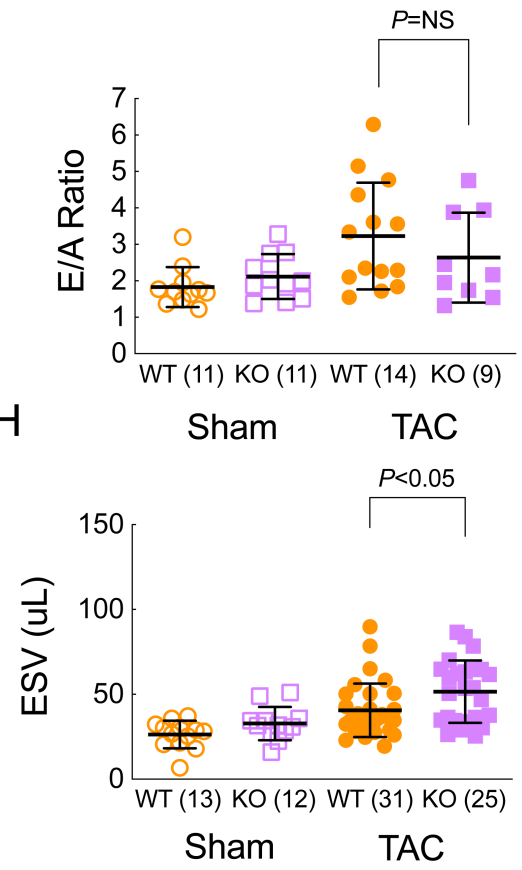

Figure 2. Cardiac function measured by echocardiography in male A-D, and female E-H, WT and Ffar4KO mice four-weeks post-TAC or sham surgery. Males: A, ejection fraction (EF, \%); B, E/A ratio; C, end diastolic volume (EDV, $\mu \mathrm{L}$ ) and $\mathbf{D}$, end systolic volume (ESV, $\mu \mathrm{L})$. Females: $\mathbf{E}$, ejection fraction (EF, $\%) ; \mathbf{F}, \mathrm{E} / \mathrm{A}$ ratio; $\mathbf{G}$, end diastolic volume (EDV, $\mu \mathrm{L}$ ) and $\mathbf{H}$. end systolic volume (ESV, $\mu \mathrm{L}$ ). Data were compared by a Welch's two sample t test. Error bars represent the mean with SD. 


\section{Figure 3. Ffar4 is necessary for induction of cardioprotective inflammatory and cell death genes, and specifically expression of genes associated with cPLA 2 signaling.}
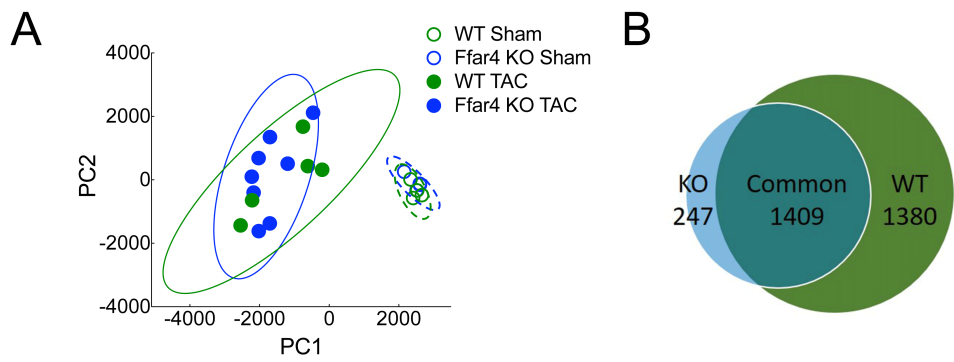

C
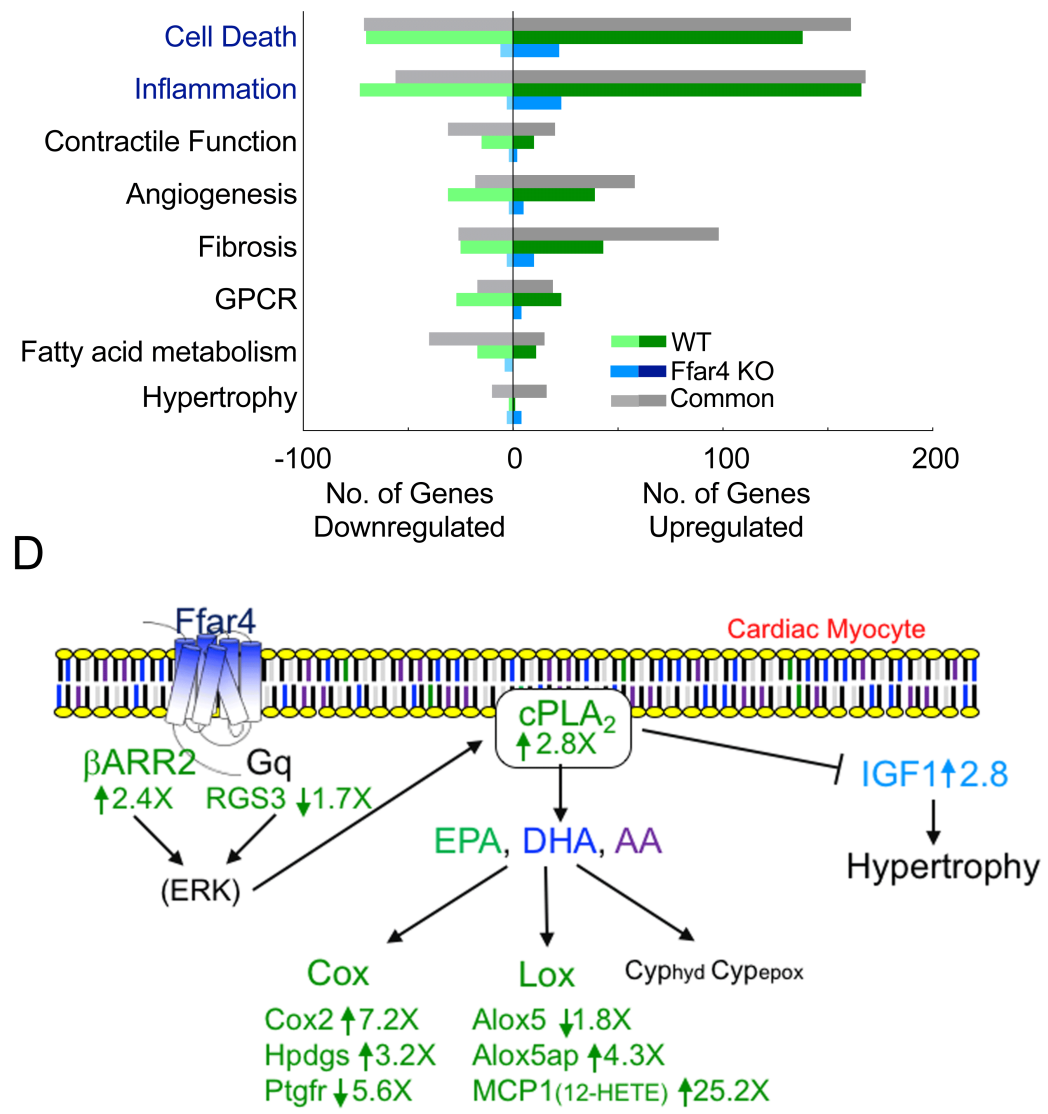

Figure 3. Transcriptome analysis (RNA-seq) was performed on cardiac myocytes isolated from male WT and Ffar4KO mice 3-days post-TAC or sham surgery. A, Principal component analysis of RNA transcriptomes from WT and Ffar4KO cardiac myocytes. B, Venn Diagram indicating genes changed $\geq$ 1.7 -fold uniquely in WT cardiac myocytes post-TAC (1,380 genes), changed $\geq 1.7$-fold uniquely in Ffar4KO cardiac myocytes post-TAC $(247$ genes $)$, or changed $\geq 1.7$-fold in both $(1,409)$. C, Differentially expressed genes represented in B were sorted based on gene ontology (GO) terms for biological function associated with eight categories (cell death, inflammation, contractile function, angiogenesis, fibrosis, GPCR, fatty acid metabolism, and hypertrophy). Graphical representation of the number of genes up- or downregulated in each category that were unique to the WT TAC (relative to sham, green), unique to Ffar4KO TAC (relative to sham, blue), and shared between WT and Ffar4KO (grey). D, Known Ffar4 signaling pathways with relevant differentially expressed genes with fold changes displayed. Genes shown in green are changed in WT TAC animals relative to WT sham; genes in blue are changed in Ffar4KO TAC compared to Ffar4KO sham animals. 
Table 1:Transcriptome analysis:

\begin{tabular}{|c|c|c|c|}
\hline GENE & GENE NAME & FOLD $\Delta$ & FUNCTION IN HEART \\
\hline \multicolumn{4}{|c|}{ CELL DEATH AND INFLAMMATION } \\
\hline IIrn1 & $\begin{array}{l}\mathrm{IL}-1 \beta \text { receptor } \\
\text { antagonist }\end{array}$ & $+220 \mathrm{WT}$ & $\begin{array}{l}\text { Blocks IL } 1 \beta \text {-receptor signaling } \\
\text { IL1 } \beta \text { a new target in HF }(60,61) \\
\text { Cantos trial shows positive results } \\
\text { post-MI with IL-1 } \beta \text {-antibody }(5)\end{array}$ \\
\hline Hmox1 & Heme-oxygenase 1 & $+24 \mathrm{WT}$ & $\begin{array}{l}\text { Cell survival/Cardioprotective } \\
\text { (Review: (62)) }\end{array}$ \\
\hline II33 & Interleukin 33 & $+4.0 \mathrm{WT}$ & Cardioprotective (Review: (62)) \\
\hline Cc/2 & $\begin{array}{l}\text { Chemokine (C-C } \\
\text { motif) ligand } 2\end{array}$ & $+25 \mathrm{WT}$ & Macrophage chemotractant \\
\hline Ccr2 & $\begin{array}{l}\text { Chemokine }(\mathrm{C}-\mathrm{C} \\
\text { motif) receptor } 2\end{array}$ & $+14 \mathrm{WT}$ & Ccl2 receptor \\
\hline Cx3cr1 & $\begin{array}{l}\text { Chemokine }(\mathrm{C}-\mathrm{X} 3-\mathrm{C} \\
\text { motif) receptor } 1\end{array}$ & $+2.8 \mathrm{WT}$ & \\
\hline \multicolumn{4}{|c|}{ Ffar4-cPLA2 SIGNALING } \\
\hline Pla2g4a & $\begin{array}{l}\text { Phospholipase A2, } \\
\text { group IVA (cytosolic, } \\
\text { calcium dependent) }\end{array}$ & $+2.8 \mathrm{WT}$ & $\begin{array}{l}\text { Activated by Ffar4 - cleaves AA or } \\
\text { EPA from membrane, substrates } \\
\text { for Cox2 } \\
\text { cPLA2 inhibits hypertrophy ( } 43) \text {. } \\
\text { Cardioprotective in ischemia ( } 44) \text {, } \\
\text { but controversial (44) }\end{array}$ \\
\hline Arrb2 & $\beta$-Aresstin2 & $+2.5 \mathrm{WT}$ & Ffar4 signals through $\beta$-Arr 2 (10) \\
\hline RGS3 & $\begin{array}{l}\text { Regulator of G-protein } \\
\text { signaling }\end{array}$ & $-1.7 \mathrm{WT}$ & \\
\hline Alox5ap & $\begin{array}{l}\text { Arachidonate } 5- \\
\text { lipoxygenase } \\
\text { activating protein }\end{array}$ & $+4.3 \mathrm{WT}$ & $\begin{array}{l}\text { Activation of 5-lipoxygenase - } \\
\text { enhanced production of } 5 \text {-HETEs }\end{array}$ \\
\hline Alox5 & $\begin{array}{l}\text { Arachidonate 5- } \\
\text { lipoxygenase }\end{array}$ & $-1.8 \mathrm{WT}$ & Production of 5 -HETEs \\
\hline Ptgs2 & Cyclooxygenase 2 & $+7.2 \mathrm{WT}$ & Prostaglandin synthesis, \\
\hline Hpgds & $\begin{array}{l}\text { Hematopoietic } \\
\text { prostaglandin D } \\
\text { synthase }\end{array}$ & $\begin{array}{l}+3.2 \mathrm{WT} \\
-5.6 \mathrm{WT}\end{array}$ & Cardioprotective (Review: (63)) \\
\hline Ptgfr & $\begin{array}{l}\text { Prostaglandin F } \\
\text { receptor }\end{array}$ & & \\
\hline Igf1 & $\begin{array}{l}\text { Insulin-like growth } \\
\text { factor } 1\end{array}$ & $+2.8 \mathrm{KO}$ & $\begin{array}{l}\text { Pro-hypertrophic, related to } \\
\text { decreased cPLA2 activity (43) }\end{array}$ \\
\hline
\end{tabular}


Figure 4. Ffar4 agonist TUG-891 increases production of 18-HEPE in adult cardiac myocytes.

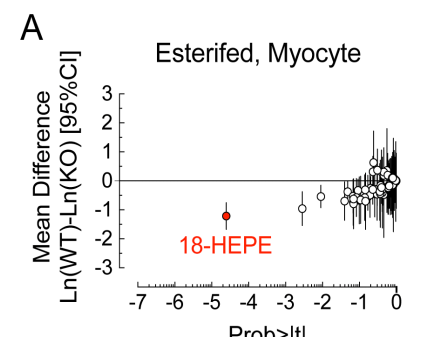

$\mathrm{E}$

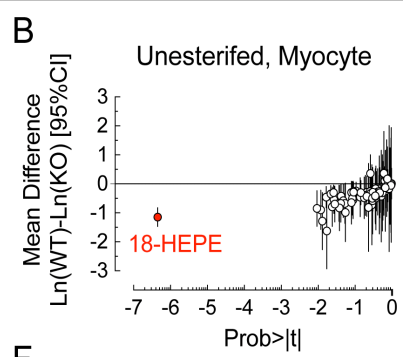

F

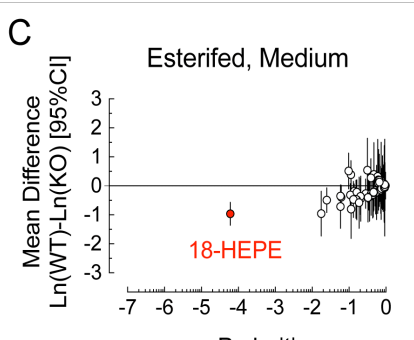

G

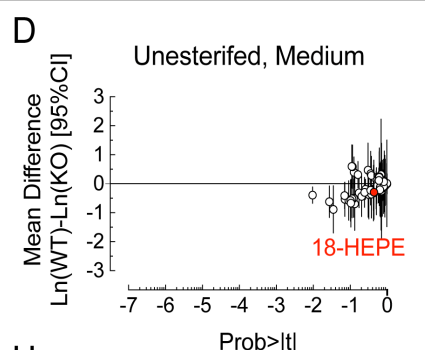

$\mathrm{H}$

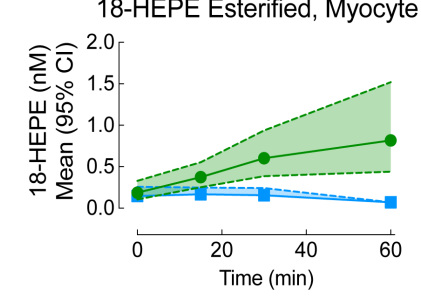

I

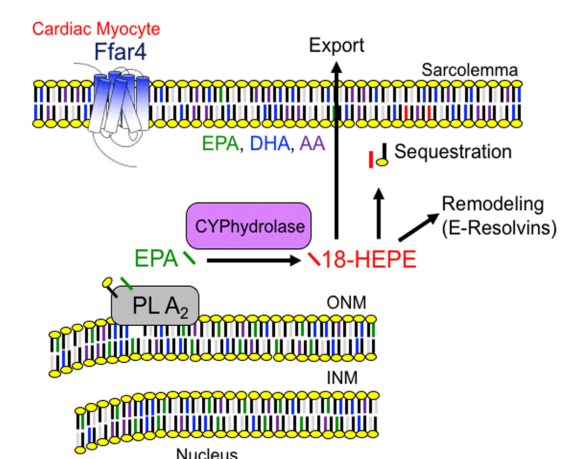

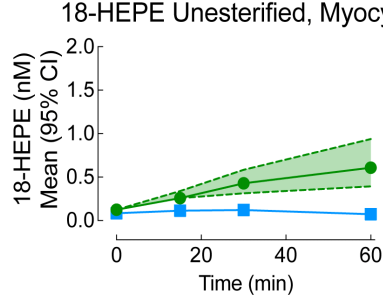

$J$

18-HEPE Esterified, Medium 18-HEPE Unsterified, Medium
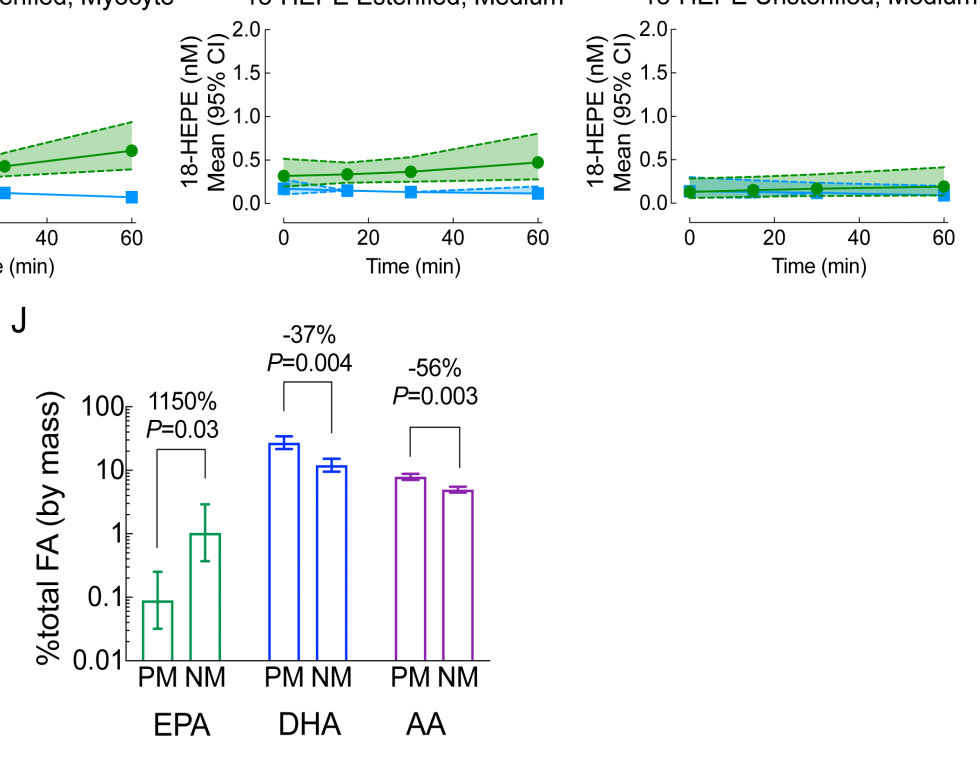

Figure 4. A-H, Cultured adult cardiac myocytes from WT (green) and Ffar4KO (blue) male mice were treated with the Ffar4 agonist, TUG891 $(50 \mu \mathrm{M})$ for $0,15,30$, and 60 minutes. Oxylipins were detected by mass spectrometry from cardiac myocyte membranes (esterified) or cytosolic fractions (non-esterified, or from the culture medium in lipoproteins (esterified) or free (non-esterified). Probability plots for oxylipins detected from cardiac myocytes in the $\mathbf{A}$, esterified or $\mathbf{B}$, unesterified fractions after 60 minutes, or in the culture medium in the $\mathbf{C}$, esterified or $\mathbf{D}$, unesterifed fractions, or time course of 18-HEPE production in myocytes in the $\mathbf{E}$, esterified or $\mathbf{F}$, unesterifed fractions, or in the medium in the $\mathbf{G}$, esterified or $\mathbf{H}$, unesterified fractions (dashed lines represent the $95 \% \mathrm{Cl}$ ). I, Cytoplasmic phospholipase $\mathrm{A}_{2} \alpha(\mathrm{cPLA} 2 \alpha)$ mediated cleavage of EPA from membrane phospholipids and production of 18-HEPE by CYPhydrolase. 18-HEPE is re-acylated into membrane phospholipids (sequestration), remains free in the cell and is further metabolized (potentially E-Resolvins), or exported, esterified in a lipoprotein. J, EPA, DHA, and AA concentration in the nuclear membrane (NM) or sarcolemma/plasma membrane (PM). Data are Mean \pm SD, $n=3$ separate preparations, fold differences indicated. 
Figure 5. Loss of Ffar4 changes circulating oxylipin content consistent with a loss of anti-inflammatory oxylipins at baseline, and prevents initiation of both mediators of inflammation and of resolution following TAC.

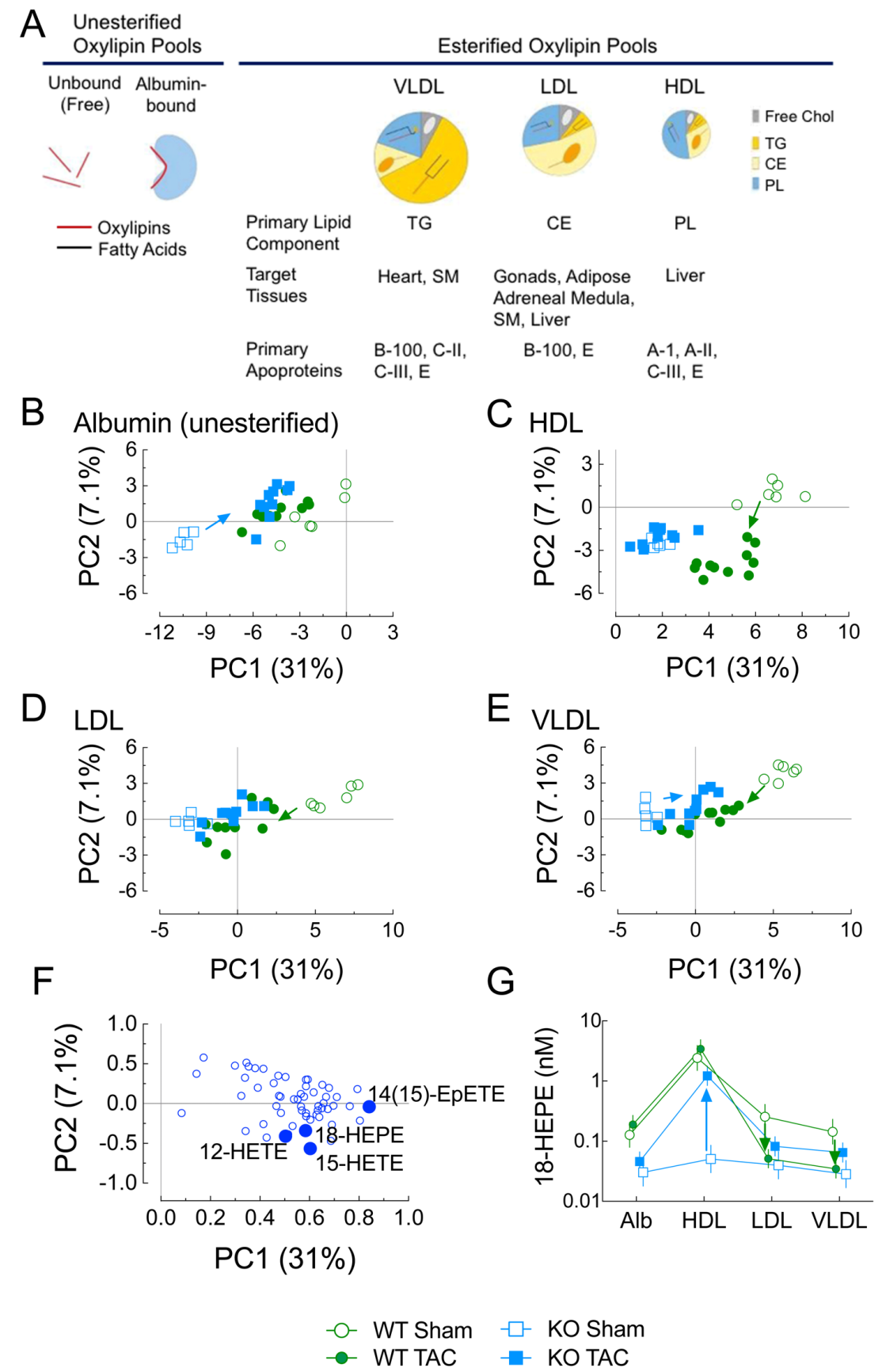

Figure 5. A. Description of plasma fractions containing unesterified oxylipins (Albumin fraction) and esterified oxylipins (Lipoprotein Fraction). TG, triglyceride; CE, cholesterol esters; PL, phospholipids; SM, skeletal muscle. B-E, Plasma was collected 4 weeks following TAC or sham surgery, and oxylipins were detected by mass spectrometry in the four plasma fractions albumin (free, unesterified fatty acids), high density lipoproteins (HDL), low-density lipoproteins (LDL) and very-low density lipoproteins (VLDL). To discriminate changes in oxylipin content between WT and Ffar4KO in sham and TAC operated mice, principle component analysis was performed for each fraction. B, Albumin, C, HDL D, LDL and E, VLDL, and F, PCA loadings. G, Specific analysis of 18-HEPE in the different plasma fractions. 
Figure 6. Ffar4 is expressed in the human heart and downregulated in $\mathrm{HF}$, with reciprocal upregulation of Ffar1.

Ffar4

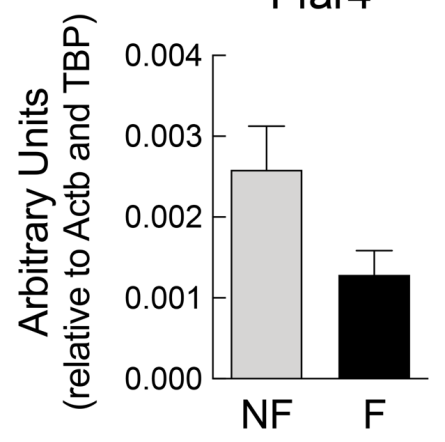

Ffar4L

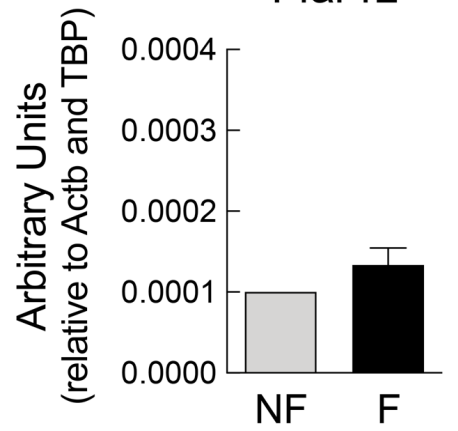

Ffar1

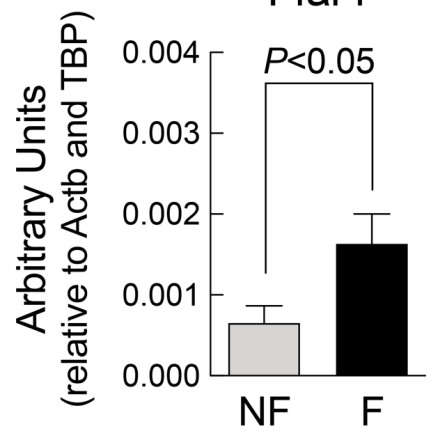

Figure 6. RT-PCR to detect expression of Ffar4 (both short and long isoforms) and Ffar1 in cardiac tissue obtained from non-failing (NF) and failing (F) human hearts. 